\title{
Graph Embedded Nonparametric Mutual Information For Supervised Dimensionality Reduction
}

\author{
Dimitrios Bouzas, Nikolaos Arvanitopoulos, Student Member, IEEE, and Anastasios Tefas, Member, IEEE
}

\begin{abstract}
In this paper, we propose a novel algorithm for dimensionality reduction that uses as a criterion the mutual information (MI) between the transformed data and their corresponding class labels. The $\mathrm{MI}$ is a powerful criterion that can be used as a proxy to the Bayes error rate. Furthermore, recent quadratic nonparametric implementations of $\mathrm{MI}$ are computationally efficient and do not require any prior assumptions about the class densities. We show that the quadratic nonparametric MI can be formulated as a kernel objective in the graph embedding framework. Moreover, we propose its linear equivalent as a novel linear dimensionality reduction algorithm. The derived methods are compared against the state-of-the-art dimensionality reduction algorithms with various classifiers and on various benchmark and real-life datasets. The experimental results show that nonparametric $M I$ as an optimization objective for dimensionality reduction gives comparable and in most of the cases better results compared with other dimensionality reduction methods.
\end{abstract}

Index Terms-Data visualization, dimensionality reduction, face recognition, feature extraction, graph embedding framework, mutual information (MI), quadratic mutual information.

\section{INTRODUCTION}

$\mathbf{T}$ HE problem of dimensionality reduction has attracted the attention of a vast number of researchers in computer vision and pattern recognition. This is mainly attributed to the fact that in many systems, dimensionality reduction is the necessary preprocessing step to efficiently manipulate highdimensional data or to denoise them [4]. A dimensionality reduction algorithm is an approach that given a set of high-dimensional data of dimensionality $d$ maps them into a lower-dimensional space of dimensionality $l$, where $l \ll d$. For this to be accomplished, many linear and nonlinear algorithms have been proposed. Among them, principal component analysis (PCA) [18] and linear discriminant analysis (LDA) [9], [12] are the most popular linear ones in the

Manuscript received August 24, 2012; revised January 18, 2014; accepted May 25, 2014. Date of publication June 24, 2014; date of current version April 15, 2015.

D. Bouzas is with Beta CAE systems S.A, Epanomi GR-57500, Greece (e-mail: bouzas@beta-cae.gr).

N. Arvanitopoulos is with the School of Computer and Communication Sciences, École Polytechnique Fédérale de Lausanne, Lausanne 1015, Switzerland (e-mail: nick.arvanitopoulos@epfl.ch).

A. Tefas is with the Department of Informatics, Aristotle University of Thessaloniki, Thessaloniki 54124, Greece (e-mail: tefas@aiia.csd.auth.gr).

Color versions of one or more of the figures in this paper are available online at http://ieeexplore.ieee.org.

Digital Object Identifier 10.1109/TNNLS.2014.2329240 categories of unsupervised and supervised dimensionality reduction, respectively. By the term supervised dimensionality reduction we mean that the specific method uses the data class labels to achieve a mapping, linear or nonlinear, such that the resulting mapped data that belong to different classes will be well separated [11]. Another linear approach, which can be either supervised or unsupervised, is the locality preserving projections (LPPs) [15]. LPP tries to preserve the local relationships within the data samples, and thus to reveal their overall structure. On the other side of dimensionality reduction lie the nonlinear methods like Laplacian eigenmaps [3], locally linear embedding [27], and ISOMAP [32]. These methods try to find nonlinear projections of the data that are more likely to detect their latent nonlinear manifold structure. With the recent advent of the kernel trick [31] most of the linear methods can be reformulated as kernel ones. Kernels have been extensively used in the context of support vectors machines (SVMs) [6]. The main idea behind the kernels is to employ a linear mapping $\phi: \mathbb{R}^{m} \rightarrow \mathcal{H}$ in a high-dimensional Hilbert space $\mathcal{H}$, where in the original space this mapping will be nonlinear [17]. Many linear methods have been extended to their kernel equivalent like kernel PCA (KPCA) [30], kernel Fisher discriminant (KFD) [26], generalized discriminant analysis (GDA) [1], complete KFD (CKFD) [36], and many recent ones like kernel orthogonal neighborhood preserving projections [22].

Recently, Yan et al. [35] proposed a general framework for dimensionality reduction, the so-called graph embedding framework. Graph embedding unifies most of the popular dimensionality reduction methods under a well-defined framework, while it gives the ability to formulate new ones. Under the context of the graph embedding framework, we propose the formulation of a novel dimensionality reduction algorithm that uses as an optimization criterion the mutual information (MI) [7]. The intuition behind the use of the MI is the fact that it is a general criterion that can overcome limitations of the previous proposed methods. MI uses high-order statistics, and not just second-order ones as, for example, LDA. Furthermore, it can be can used as an alternative to the Bayes error rate, which is the optimal criterion for classification [33]. However, computing the MI is computationally inefficient, since probability density functions of variables are required and also high-dimensional numerical integration of thoses. In [33], an efficient quadratic nonparametric formulation of the MI (QMI) between the data feature vectors and their 
corresponding class labels is proposed, which gives better MI estimation in high-dimensional spaces with acceptable computational cost.

In our paper, we show that QMI can be integrated in the graph embedding framework and hence, we are able to derive a closed mathematical form for the optimization of the corresponding objective criterion. In particular, we show that under the graph embedding framework, QMI can be reformulated to produce a kernel dimensionality reduction method that we call kernel QMI (KQMI). Second, we derive the linear equivalent of this method, called Linear QMI (LQMI). Under the perspective of the closed form optimization formula, we are given the opportunity to evaluate the use of the nonparametric MI as a dimensionality reduction objective. Furthermore, we compare the reformulated methods against the mainstream projective dimensionality reduction approaches available, with various classifiers and on several benchmark and real-life datasets. Our results show that our proposed algorithms attain comparable and in most of the cases better results than other state-of-theart supervised dimensionality reductions methods.

Our paper is organized as follows. In Section II, we describe the prior work of several researchers that form the basis of our proposed method. In Section III, we give all the theoretical background of our paper. In Section IV, we illustrate our experimental results. Finally, in Section V, we conclude our paper.

\section{PRIOR WORK}

In this section, we comment on the work of several researchers that supplied the motivation for the derivation of our work.

\section{A. Graph Embedding Framework}

Recent work has shown that many dimensionality reduction algorithms can be reformulated into the graph embedding framework [35]. The graph embedding framework is based on the introduction of the undirected weighted graph $\mathbf{G}=(\mathbf{X}, \mathbf{W})$, with vertex set the data points $\mathbf{X}=$ $\left[\mathbf{x}_{1}, \ldots, \mathbf{x}_{n}\right]^{\mathrm{T}} \in \mathbb{R}^{n \times d}$ and similarity matrix $\mathbf{W} \in \mathbb{R}^{n \times n}$. The graph embedding of the graph $\mathbf{G}$ is, therefore, an algorithm to find the low-dimensional representation of the data that best preserve the relationships between the vertex pairs of $\mathbf{G}$. The graph $\mathbf{G}$ can be seen as an intrinsic graph. Furthermore, a penalty graph $\mathbf{G}^{p}=\left(\mathbf{X}, \mathbf{W}^{p}\right)$ can also be defined, such that the weight matrix of the graph penalizes specific characteristics of the data structure. For the 1-D case, assuming that $\mathbf{y}=\left[y_{1}, \ldots, y_{n}\right]^{\mathrm{T}}$ is the vector containing the projections of each data sample $\mathbf{x}_{i}$, the graph criterion to be optimized is

$$
\mathbf{y}^{*}=\arg \min _{\mathbf{y}^{\top} \mathbf{C y}=\epsilon} \sum_{i, j=1}^{n}\left\|y_{i}-y_{j}\right\|^{2} W_{i j}=\arg \min _{\mathbf{y}^{\top} \mathbf{C y}=\epsilon} \mathbf{y}^{\top} \mathbf{L y}
$$

where $\mathbf{L}$ is the graph Laplacian defined as $\mathbf{L}=\mathbf{D}-\mathbf{W}$ and $\mathbf{D}$ is the diagonal degree matrix defined as $D_{i i}=\sum_{j=1}^{n} W_{i j}, i=$ $1, \ldots, n$. $\mathbf{C}$ is a constraint matrix to avoid trivial solutions and is typically a diagonal matrix for scale normalization, or the graph Laplacian of $\mathbf{G}^{p}$, that is, $\mathbf{C}=\mathbf{L}^{p}=\mathbf{D}^{p}-\mathbf{W}^{p}$ and $\epsilon$ is a constant. If we assume that the vector $\mathbf{y}$ is obtained by the linear projection $\mathbf{y}=\mathbf{X w}$, where $\mathbf{w} \in \mathbb{R}^{d}$ is the projection vector, then the objective becomes

$$
\begin{aligned}
\mathbf{w}^{*} & =\arg \min _{\mathbf{w}^{\top} \mathbf{X}^{\top} \mathbf{C X} \mathbf{w}=\epsilon} \sum_{\substack{\mathbf{w}^{\top} \mathbf{w}=\epsilon \\
i, j=1}}^{n}\left\|\mathbf{w}^{\top} \mathbf{x}_{i}-\mathbf{w}^{\top} \mathbf{x}_{j}\right\|^{2} W_{i j} \\
& =\arg \underset{\substack{\mathbf{w}^{\top} \mathbf{X}^{\top} \mathbf{C X} \mathbf{w}=\epsilon \\
\text { or } \mathbf{w}^{\top} \mathbf{w}=\epsilon}}{\min } \mathbf{w}^{\top} \mathbf{X}^{\top} \mathbf{L X} \mathbf{X} .
\end{aligned}
$$

Following similar arguments to [30] in the case of PCA (see also (4) below) we see that the solution to the objective (2) should lie on the span of the data points $\mathbf{x}_{1}, \ldots, \mathbf{x}_{n}$, therefore, it can be written as a linear combination of the form $\mathbf{w}=\sum_{i=1}^{n} \beta_{i} \mathbf{x}_{i}$.

The objective in (2), even though is computationally efficient to optimize, is not always optimal in terms of classification performance, especially when the underlying data are distributed in a highly nonlinear way. A solution to this problem is to introduce the kernel extension of objective (2) to handle nonlinearly distributed data using the kernel trick [31]. The input data are mapped to a higher dimensional Hilbert space $\mathcal{H}$ using a map $\phi: \mathbf{x} \rightarrow \mathcal{H}$. In this new feature space, a linear projection algorithm is performed similar to (2). The key property of the kernel trick is that it is based only on inner products of data pairs defined by the kernel function $k\left(\mathbf{x}_{i}, \mathbf{x}_{j}\right)=\phi\left(\mathbf{x}_{i}\right)^{\top} \phi\left(\mathbf{x}_{j}\right)$. As in the linear case, the projection direction $\mathbf{w} \in \mathbb{R}^{n}$ lies in the span of $\phi\left(\mathbf{x}_{1}\right), \ldots, \phi\left(\mathbf{x}_{n}\right)$ (see also [30]), therefore, it admits a representation of the form $\mathbf{w}=\sum_{i=1}^{n} \alpha_{i} \phi\left(\mathbf{x}_{i}\right)$. By defining the kernel matrix $\mathbf{K} \in \mathbb{R}^{n \times n}$ as $K_{i j}=\phi\left(\mathbf{x}_{i}\right)^{\top} \phi\left(\mathbf{x}_{j}\right)$, the objective in (2) can be written as

$$
\begin{aligned}
& \boldsymbol{\alpha}^{*}=\arg \min _{\substack{\boldsymbol{\alpha}^{\top} \mathbf{K}^{\top} \mathbf{C K} \boldsymbol{\alpha}=\epsilon \\
\text { or } \boldsymbol{\alpha}^{\top} \mathbf{K} \boldsymbol{\alpha}=\epsilon}} \sum_{i, j=1}^{n}\left\|\boldsymbol{\alpha}^{\top} \mathbf{K}_{i}-\boldsymbol{\alpha}^{\top} \mathbf{K}_{j}\right\|^{2} W_{i j} \\
& =\arg \min _{\substack{\boldsymbol{\alpha}^{\top} \mathbf{K}^{\top} \mathbf{C K} \boldsymbol{\alpha}=\epsilon \\
\text { or } \boldsymbol{\alpha}^{\top} \mathbf{K} \boldsymbol{\alpha}=\epsilon}} \boldsymbol{\alpha}^{\top} \mathbf{K}^{\top} \mathbf{L K} \boldsymbol{\alpha} .
\end{aligned}
$$

The solutions of (1)-(3) can be obtained by solving the generalized eigenvalue problem

$$
\mathbf{A v}=\lambda \mathbf{B v}
$$

where, $\mathbf{A}=\mathbf{L}, \mathbf{X}^{\top} \mathbf{L X}, \mathbf{K}^{\top} \mathbf{L K}$ and $\mathbf{B}=\mathbf{I}, \mathbf{C}, \mathbf{X}^{\top} \mathbf{C X}$, $\mathbf{K}, \mathbf{K}^{\top} \mathbf{C K}$, depending on the type of the problem.

\section{B. Quadratic Mutual Information}

Many ways have been proposed to accurately estimate the MI between data points and their respective class labels. QMI [33] is an accurate estimation method for highdimensional problems with acceptable computational cost. We assume a random variable $X$ representing the data points $\mathbf{x}_{i} \in \mathbb{R}^{d}$ and a discrete random variable $Y$ representing the class labels. Therefore, we have data pairs of the form $\left\{\mathbf{x}_{i}, y_{i}\right\}_{i=1}^{n}$. Let $p(\mathbf{x})$ be the probability density function of the data points and $P(Y)$ the class prior probabilities. The MI between the two random variables is defined as

$$
\mathcal{I}(X, Y)=\sum_{y} \int_{\mathbf{x}} p(\mathbf{x}, y) \log \frac{p(\mathbf{x}, y)}{p(\mathbf{x}) P(y)} d \mathbf{x} .
$$


The MI is a measure of dependence between random variables, in our case between the data points $X$ and their class labels $Y$. The above equation can also be interpreted as a Kullback-Leibler divergence

$\mathcal{K} \mathcal{L}\left(Q_{1}(\mathbf{x}, y), Q_{2}(\mathbf{x}, y)\right)=\sum_{y} \int_{\mathbf{x}} Q_{1}(\mathbf{x}, y) \log \frac{Q_{1}(\mathbf{x}, y)}{Q_{2}(\mathbf{x}, y)} d \mathbf{x}$

where $Q_{1}(\mathbf{x}, y)=p(\mathbf{x}, y)$ and $Q_{2}(\mathbf{x}, y)=p(\mathbf{x}) P(y)$. In [20, p.178] and [21, Ch. 4], it is argued that if our goal is to find the distribution that maximizes the divergence and not to compute its absolute value, the axioms used in deriving divergence measures can be relaxed resulting in the same maximizing distribution. One such measure that satisfies the relaxed axioms is given by

$D_{\alpha}\left(Q_{1}, Q_{2}\right)=\frac{1}{\alpha(\alpha-1)} \sum_{i=1}^{n}\left(q_{1, i}^{\alpha}-\alpha q_{1, i} q_{2, i}^{\alpha-1}+(\alpha-1) q_{2, i}^{\alpha}\right)$

where $\alpha \neq 0,1$. Selecting $\alpha=2$ and extending the measure to continuous densities we arrive at the quadratic measure given by (up to a constant)

$$
D_{2}\left(Q_{1}, Q_{2}\right)=\int_{\mathbf{x}}\left(Q_{1}(\mathbf{x})-Q_{2}(\mathbf{x})\right)^{2} d \mathbf{x} .
$$

Another justification for using the quadratic divergence measure is given in [33], where it is shown that maximizing $D_{2}\left(Q_{1}, Q_{2}\right)$ is equivalent to maximizing a lower bound to $\mathcal{K} \mathcal{L}\left(Q_{1}, Q_{2}\right)$. The MI can now be expressed in terms of the divergence between the joint density and the product of its marginals. Inserting these forms of the distributions into (8) leads to the QMI measure between two continuous variables $\mathbf{x}_{1}, \mathbf{x}_{2}$

$$
\mathcal{I}_{\mathcal{Q}}\left(X_{1}, X_{2}\right)=\int_{\mathbf{x}_{1}} \int_{\mathbf{x}_{2}}\left(p\left(\mathbf{x}_{1}, \mathbf{x}_{2}\right)-p\left(\mathbf{x}_{1}\right) p\left(\mathbf{x}_{2}\right)\right)^{2} d \mathbf{x}_{1} \mathbf{x}_{2} .
$$

In our case, the QMI between the continuous variable $X$ of the data points and the discrete random variable $Y$ of the class labels is defined as

$$
\begin{aligned}
\mathcal{I}_{\mathcal{Q}}(X, Y)= & \sum_{y} \int_{\mathbf{x}} p(\mathbf{x}, y)^{2} d \mathbf{x}+\sum_{y} \int_{\mathbf{x}} p(\mathbf{x})^{2} P(y)^{2} d \mathbf{x} \\
& -2 \sum_{y} \int_{\mathbf{x}} p(\mathbf{x}, y) p(\mathbf{x}) P(y) d \mathbf{x} .
\end{aligned}
$$

In [33], the above probability distributions are approximated using Parzen window estimators with a Gaussian kernel. Therefore, $p(\mathbf{x}), P\left(y_{i}=c\right)$ and $p\left(\mathbf{x}, y_{i}=c\right)$ can be written as

$$
\begin{aligned}
p(\mathbf{x}) & =\sum_{y} p(\mathbf{x}, y)=\frac{1}{n} \sum_{c=1}^{C} \sum_{j=1}^{J_{c}} \mathcal{N}\left(\mathbf{x} ; \mathbf{x}_{j}, \sigma^{2} I\right) \\
& =\frac{1}{n} \sum_{i=1}^{n} \mathcal{N}\left(\mathbf{x} ; \mathbf{x}_{i}, \sigma^{2} \mathbf{I}\right) \\
P\left(y_{i}=c\right) & =\frac{J_{c}}{n} \\
p\left(\mathbf{x} \mid y_{i}=c\right) & =\frac{1}{J_{c}} \sum_{i: y_{i}=c} \mathcal{N}\left(\mathbf{x} ; \mathbf{x}_{i}, \sigma^{2} \mathbf{I}\right)
\end{aligned}
$$

where in the above equations $n$ is the total number of data points, $C$ is the total number of classes, $J_{c}$ is the number of samples of class $c$, and $\mathcal{N}(\mathbf{x} ; \mathbf{m}, \Sigma)$ denotes the Gaussian probability distribution function with mean vector $\mathbf{m}$ and covariance matrix $\Sigma$, defined as

$\mathcal{N}(\mathbf{x} ; \mathbf{m}, \Sigma)=\frac{1}{\sqrt{2 \pi|\Sigma|}} \exp \left(-\frac{1}{2}(\mathbf{x}-\mathbf{m})^{\top} \Sigma^{-1}(\mathbf{x}-\mathbf{m})\right)$.

By expanding (10) we get the following equation:

$$
\mathcal{I}_{Q}(\mathbf{x}, y)=V_{\mathrm{IN}}+V_{\mathrm{ALL}}-2 V_{\mathrm{BTW}}
$$

where

$$
\begin{aligned}
V_{\mathrm{IN}} & =\sum_{y} \int_{\mathbf{x}} p(\mathbf{x}, y)^{2} d \mathbf{x} \\
& =\frac{1}{n^{2}} \sum_{c=1}^{C} \sum_{j: y_{j}=c} \sum_{k: y_{k}=c} \mathcal{N}\left(\mathbf{x}_{j}-\mathbf{x}_{k} ; \mathbf{0}, 2 \sigma^{2} \mathbf{I}\right) \\
V_{\mathrm{ALL}} & =\sum_{y} \int_{\mathbf{x}} p(\mathbf{x})^{2} P(y)^{2} d \mathbf{x} \\
& =\frac{1}{n^{2}} \sum_{c=1}^{C}\left(\frac{J_{c}}{N}\right)^{2} \sum_{j=1}^{n} \sum_{k=1}^{n} \mathcal{N}\left(\mathbf{x}_{j}-\mathbf{x}_{k} ; \mathbf{0}, 2 \sigma^{2} \mathbf{I}\right) \\
V_{\mathrm{BTW}} & =\sum_{y} \int_{\mathbf{x}} p(\mathbf{x}, y) p(\mathbf{x}) P(y) d \mathbf{x} \\
& =\frac{1}{n^{2}} \sum_{c=1}^{C} \frac{J_{c}}{n} \sum_{j=1}^{n} \sum_{k: y_{k}=c} \mathcal{N}\left(\mathbf{x}_{l}-\mathbf{x}_{k} ; \mathbf{0}, 2 \sigma^{2} \mathbf{I}\right) .
\end{aligned}
$$

The above pairwise interactions between samples, $V_{\mathrm{IN}}, V_{\mathrm{ALL}}$, and $V_{\mathrm{BTW}}$ can be interpreted as follows [33].

1) $V_{\mathrm{IN}}$ can be seen as interactions between pairs of samples inside each class.

2) $V_{\text {ALL }}$ consists of interactions between all pairs of samples, regardless of class membership.

3) $V_{\text {BTW }}$ consists of interactions between samples of each class against all other samples.

\section{GRAPH EMBEDDING OF QMI}

In this section, we show that the QMI can be formulated into the graph embedding framework and can be interpreted as a direct kernelization of a linear objective.

\section{A. Formulation of KQMI and LQMI Algorithms}

We assume our initial data points are centralized, that is they have zero mean. Otherwise, we subtract from each data sample the mean vector of the whole dataset. We define the centralized kernel matrix $\mathbf{K} \in \mathbb{R}^{n \times n}$ with elements $K_{i j}=$ $\left(\widetilde{\mathbf{K}}-\mathbf{E}_{n} \widetilde{\mathbf{K}}-\widetilde{\mathbf{K}} \mathbf{E}_{n}+\mathbf{E}_{n} \widetilde{\mathbf{K}} \mathbf{E}_{n}\right)_{i j}$, where $\widetilde{K}_{i j}=\mathcal{N}\left(\mathbf{x}_{i}-\mathbf{x}_{j}\right.$; $\left.\mathbf{0}, 2 \sigma^{2} \mathbf{I}\right)$ and $\mathbf{E}_{n}$ the $n \times n$ matrix with all elements equal to $1 / n$. The kernel matrix can also be written as $\mathbf{K}=\Phi \Phi^{\top}$, where $\Phi \in \mathbb{R}^{n \times m}$ is the matrix of the mapped data points $\mathbf{X} \in$ $\mathcal{X}$ into a Hilbert space $\mathcal{H}$ through the mapping $\phi: \mathcal{X} \rightarrow \mathcal{H}$ and $m$ is the unknown dimensionality of the feature space. We define $\mathbf{1}=[1, \ldots, 1]^{\top} \in \mathbb{R}^{n}$ and $\mathbf{1}_{c} \in \mathbb{R}^{n}$ with elements

$$
\left[\mathbf{1}_{c}\right]_{i}= \begin{cases}1 & \text { if } \mathbf{x}_{i} \in c \\ 0 & \text { else }\end{cases}
$$


Furthermore, we define the constants $C_{\mathrm{ALL}}=$ $1 / n^{4} \sum_{c=1}^{C}\left(J_{c}\right)^{2}, C_{\mathrm{IN}}=1 / n^{2}$, and $C_{\mathrm{BTW}, c}=J_{c} / n^{3}, \quad c=$ $1, \ldots, C$. With the above notation, the $V_{\mathrm{ALL}}, V_{\mathrm{IN}}$, and $V_{\mathrm{BTW}}$ terms can be written as

$$
\begin{aligned}
V_{\mathrm{ALL}} & =C_{\mathrm{ALL}} \operatorname{tr}\left\{\Phi^{\top} \mathbf{1 1}^{\top} \Phi\right\} \\
V_{\mathrm{IN}} & =C_{\mathrm{IN}} \operatorname{tr}\left\{\Phi^{\top}\left(\sum_{c=1}^{C} \mathbf{1}_{c} \mathbf{1}_{c}^{\top}\right) \Phi\right\} \\
V_{\mathrm{BTW}} & =\operatorname{tr}\left\{\Phi^{\top} \mathbf{1}\left(\sum_{c=1}^{C} C_{\mathrm{BTW}, c} \mathbf{1}_{c}^{\top}\right) \Phi\right\} .
\end{aligned}
$$

Using the above, the QMI between the data points and their respective class labels can be reformulated as

$$
\begin{array}{r}
\mathcal{I}_{\mathcal{Q}}(\mathbf{x}, y)=\operatorname{tr}\left\{\Phi ^ { \top } \left(C_{\mathrm{ALL}} \mathbf{1 1}^{\top}+C_{\mathrm{IN}}\left(\sum_{c=1}^{C} \mathbf{1}_{c} \mathbf{1}_{c}^{\top}\right)\right.\right. \\
\left.\left.-2 \cdot \mathbf{1}\left(\sum_{c=1}^{C} C_{\mathrm{BTW}, c} \mathbf{1}_{c}^{\top}\right)\right) \Phi\right\} .
\end{array}
$$

Detailed derivations of the expressions (15)-(17) can be found in Appendix A. To compute nonlinear projections of the data points, we define the projection vectors $\mathbf{w}_{i} \in \mathbb{R}^{m}, i=$ $1, \ldots, n$. We can restrict these vectors to be in the range of $\Phi$, since they belong to $\mathbb{R}^{m}$, which is the column space of $\Phi$. Therefore, they can admit a representation of the form $\mathbf{w}_{i}=\sum_{j}^{n} \alpha_{i j} \phi\left(\mathbf{x}_{j}\right)=\Phi^{\top} \boldsymbol{\alpha}_{i}$. By arranging them as columns of a matrix we can create the projection matrix $\mathbf{W}=\left\{\mathbf{w}_{i}\right\}_{i=1}^{n}=\left\{\Phi^{\top} \boldsymbol{\alpha}_{i}\right\}_{i=1}^{n}=\Phi^{\top} \mathbf{A} \in \mathbb{R}^{n \times n}$, where $\mathbf{A}=$ $\left\{\boldsymbol{\alpha}_{i}\right\}_{i=1}^{n} \in \mathbb{R}^{n \times n}$. After the nonlinear projection with the matrix $\mathbf{W}$ and by defining

$\mathbf{M}=\left(C_{\mathrm{ALL}} \mathbf{1} \mathbf{1}^{\top}+C_{\mathrm{IN}}\left(\sum_{c=1}^{C} \mathbf{1}_{c} \mathbf{1}_{c}^{\top}\right)-2 \cdot \mathbf{1}\left(\sum_{c=1}^{C} C_{\mathrm{BTW}, c} \mathbf{1}_{c}^{\top}\right)\right)$

we result in the following formulation of the QMI inspired graph embedding objective:

$$
\mathcal{I}_{\mathcal{Q}}^{\mathcal{P}}(\mathbf{x}, y)=\operatorname{tr}\left\{(\Phi \mathbf{W})^{\top} \mathbf{M} \Phi \mathbf{W}\right\}=\operatorname{tr}\left\{\mathbf{A}^{\top} \mathbf{K M K A}\right\} .
$$

The above formulation uses the matrix $\mathbf{M}$ that represents the interactions between data samples in the graph embedding framework and has been extracted by the QMI formulation in (18) to connect the data samples after projection to an arbitrary Hilbert space (represented in $\Phi^{\top}$ ) and dimensionality reduction (represented in $\left.(\Phi \mathbf{W})^{\top}=\mathbf{A}^{\top} \mathbf{K}\right)$. The optimal matrix $\mathbf{A}$ can be computed by solving the following optimization problem:

$$
\mathbf{A}^{*}=\arg \max _{\mathbf{A}^{\top} \mathbf{K} \mathbf{A}=\mathbf{I}} \operatorname{tr}\left\{\mathbf{A}^{\top} \mathbf{K} \mathbf{M K} \mathbf{A}\right\} .
$$

The constraint $\mathbf{A}^{\top} \mathbf{K A}=\mathbf{I}$ is derived from the orthogonality constraint of the projection matrix $\mathbf{W}$, that is, $\mathbf{W}^{\top} \mathbf{W}=\mathbf{I} \Rightarrow$ $\left(\Phi^{\top} \mathbf{A}\right)^{\top}\left(\Phi^{\top} \mathbf{A}\right)=\mathbf{I} \Rightarrow \mathbf{A}^{\top} \Phi \Phi^{\top} \mathbf{A}=\mathbf{I} \Rightarrow \mathbf{A}^{\top} \mathbf{K} \mathbf{A}=\mathbf{I}$. In general, the matrix $\mathbf{M}$ is not symmetric due to the $C_{\mathrm{BTW}, c}$ terms, unless the classes of the dataset are balanced. However, it is known that for every general square matrix $\mathbf{M} \in \mathbb{R}^{n \times n}$ and $\mathbf{x} \in \mathbb{R}^{n}$, it holds $\mathbf{x}^{\top} \mathbf{M x}=\mathbf{x}^{\top} \mathbf{M}^{\prime} \mathbf{x}$, where $\mathbf{M}^{\prime}=\left(\mathbf{M}+\mathbf{M}^{\top}\right) / 2$ the symmetrization of $\mathbf{M}$ [14]. By symmetrizing the matrix $\mathbf{M}$ in (19) we obtain the equivalent objective

$$
\mathbf{A}^{*}=\arg \max _{\mathbf{A}^{\top} \mathbf{K A}=\mathbf{I}} \operatorname{tr}\left\{\mathbf{A}^{\top} \mathbf{K} \mathbf{M}^{\prime} \mathbf{K} \mathbf{A}\right\} .
$$

To make the problem well-posed (see also Section III-C), we additionally enforce the constraint that the embedding vectors have unit covariance, that is, $(1 / n)(\Phi \mathbf{W})^{\top}(\Phi \mathbf{W})=$ $\mathbf{I} \Rightarrow \frac{1}{n}\left(\Phi \Phi^{\top} \mathbf{A}\right)^{\top}\left(\Phi \Phi^{\top} \mathbf{A}\right)=\mathbf{I} \Rightarrow(\mathbf{K A})^{\top}(\mathbf{K A})=n \mathbf{I} \Rightarrow$ $\mathbf{A}^{\top} \mathbf{K K A}=n \mathbf{I}$. The final objective becomes

$$
\mathbf{A}^{*}=\arg \max _{\mathbf{A}^{\top} \mathbf{K A}=\mathbf{I}} \frac{\operatorname{tr}\left\{\mathbf{A}^{\top} \mathbf{K} \mathbf{M}^{\prime} \mathbf{K} \mathbf{A}\right\}}{\operatorname{tr}\left\{\mathbf{A}^{\top} \mathbf{K} \mathbf{K} \mathbf{A}\right\}} .
$$

The solution of the above optimization problem is given by the generalized eigenvalue problem

$$
\mathbf{K M}^{\prime} \mathbf{K U}=\Lambda \mathbf{K K U} \Leftrightarrow \mathbf{M}^{\prime} \mathbf{K U}=\boldsymbol{\Lambda} \mathbf{K U}
$$

where $\boldsymbol{\Lambda}$ is the diagonal matrix of the eigenvalues $\lambda_{i}$ and $\mathbf{U}$ is the matrix whose columns contain the eigenvectors $\boldsymbol{v}_{i}$. To satisfy the constraint $\mathbf{A}^{\top} \mathbf{K A}=\mathbf{I}$, each eigenvector $\boldsymbol{v}_{i}$ must be divided by $\sqrt{\boldsymbol{v}_{i} \mathbf{K} \boldsymbol{v}_{i}}$ to get normalized eigenvectors $\boldsymbol{v}_{i}$. It is important to note here that the matrix $\mathbf{M}^{\prime}$ has rank $C-1$, therefore the product $\mathbf{K} \mathbf{M}^{\prime} \mathbf{K}$ has maximum rank also $C-1$. As a result, using this formulation, we are able to utilize only $C-1$ eigenvectors that belong to the column space of $\mathbf{K M}^{\prime} \mathbf{K}$ and the maximum projection dimension is $C-1$. The optimal nonlinear projection of the data points to dimension $l=$ $1, \ldots, C-1$ is given by the first $l$ dominant eigenvectors $\boldsymbol{v}_{i}$, $i=1, \ldots, l$, that is $\mathbf{A}^{*}=\left\{\boldsymbol{v}_{i}\right\}_{i=1}^{l}$. The matrix of the projected data points is given by $\mathbf{K}^{\mathcal{P}}=\mathbf{K} \mathbf{A}^{*}$.

Another more robust strategy to solve (23) is to apply an eigenvalue decomposition of the kernel matrix $\mathbf{K}=\mathbf{P L P}^{\top}$, where $\mathbf{P}$ is the matrix which contains in its columns the eigenvectors of $\mathbf{K}$ and $\mathbf{L}$ is the diagonal matrix that contains the eigenvalues of $\mathbf{K}$. The quotient now becomes

$$
Q=\frac{\operatorname{tr}\left\{\mathbf{A}^{\top} \mathbf{P} \mathbf{L} \mathbf{P}^{\top} \mathbf{M}^{\prime} \mathbf{P} \mathbf{L} \mathbf{P}^{\top} \mathbf{A}\right\}}{\operatorname{tr}\left\{\mathbf{A}^{\top} \mathbf{P} \mathbf{L} \mathbf{P}^{\top} \mathbf{P L} \mathbf{P}^{\top} \mathbf{A}\right\}} .
$$

By defining $\mathbf{B}=\mathbf{L} \mathbf{P}^{\top} \mathbf{A}$ and using the fact that $\mathbf{P}$ is orthonormal, the quotient becomes

$$
Q=\frac{\operatorname{tr}\left\{\mathbf{B}^{\top} \mathbf{P}^{\top} \mathbf{M}^{\prime} \mathbf{P B}\right\}}{\operatorname{tr}\left\{\mathbf{B}^{\top} \mathbf{B}\right\}} .
$$

This quotient is maximized by solving the eigenvalue problem

$$
\mathbf{P}^{\top} \mathbf{M}^{\prime} \mathbf{P Z}=\mathbf{H Z}
$$

where $\mathbf{Z}$ is the matrix containing the eigenvectors of $\mathbf{P}^{\top} \mathbf{M}^{\prime} \mathbf{P}$ and $\mathbf{H}$ a diagonal matrix containing the corresponding eigenvalues. The optimal projection vectors can now be computed by $\mathbf{A}^{*}=\mathbf{P}^{-\top} \mathbf{L}^{-1} \mathbf{B}=\mathbf{P} \mathbf{L}^{-1} \mathbf{B}$.

The complete algorithm to compute the optimal nonlinear projections produced by the QMI between the data points and their respective labels is given in Algorithm 1. The objective in (23) can be seen as a direct kernelization of a linear objective of the form

$$
\mathbf{W}^{*}=\arg \max _{\mathbf{W}^{\top} \mathbf{W}=\mathbf{I}} \frac{\operatorname{tr}\left\{\mathbf{W}^{\top} \mathbf{X}^{\top} \mathbf{M}^{\prime} \mathbf{X W}\right\}}{\operatorname{tr}\left\{\mathbf{W}^{\top} \mathbf{X}^{\top} \mathbf{X} \mathbf{W}\right\}}
$$




\section{Algorithm 1 Kernel QMI \\ Input: \\ - Vector $\mathbf{y}=\left\{y_{i} \mid i=1, \ldots, n\right\}, y_{i} \in\{1, \ldots, C\}$. \\ - Centralized data matrix $\mathbf{X}=\left(\left\{\mathbf{x}_{i}\right\}_{i=1}^{n}\right)^{\top} \in \mathbb{R}^{n \times m}$ with zero mean.}

\section{Output:}

- Non-linearly projected data $\mathbf{K}^{\mathcal{P}} \in \mathbb{R}^{n \times d}$.

\section{Step 1:}

1: Calculate centralized kernel matrix $\mathbf{K} \in \mathbb{R}^{n \times n}$,

$K_{i j}=\left(\widetilde{\mathbf{K}}-\mathbf{E}_{n} \widetilde{\mathbf{K}}-\widetilde{\mathbf{K}}_{n}+\mathbf{E}_{n} \widetilde{\mathbf{K}}_{n}\right)_{i j}$, where

$\widetilde{K}_{i j}=\mathcal{N}\left(\mathbf{x}_{i}-\mathbf{x}_{j}, \sigma^{2} \mathbf{I}\right)$ and $\mathbf{E}_{n}$ the $n \times n$ matrix with all elements equal to $1 / n$

2: Calculate matrix $\mathbf{M}$ using (19) and symmetrize it to obtain $\mathbf{M}^{\prime}$.

Step 2:

1: Decompose the kernel matrix $\mathbf{K}$ into its eigenvectors and eigenvalues:

$$
\mathbf{K}=\mathbf{P L} \mathbf{P}^{\top} \text {. }
$$

2: Solve the eigenvalue problem

$$
\mathbf{P}^{\top} \mathbf{M}^{\prime} \mathbf{P Z}=\mathbf{H Z} .
$$

where $\mathbf{H}$ is the diagonal matrix containing the eigenvalues of $\mathbf{P}^{\top} \mathbf{M}^{\prime} \mathbf{P}$ and $\mathbf{Z}$ is the matrix whose columns are the corresponding eigenvectors.

3: Sort the eigenvalues with descending order and arrange the corresponding eigenvectors into the matrix $\mathbf{B}$.

4: Compute the optimal projection vectors as

$$
\mathbf{A}=\mathbf{P} \mathbf{L}^{-1} \mathbf{B} \text {. }
$$

\section{Step 3:}

1: Select the first $d=1, \ldots, C-1$ eigenvectors of $\mathbf{A}$ to create a new matrix $\mathbf{A}^{*}$ and compute the resulting projected data as

$$
\mathbf{K}^{\mathcal{P}}=\mathbf{K A}^{*} \text {. }
$$

where $\mathbf{W}=\left\{\mathbf{w}_{i}\right\}_{i=1}^{n} \in \mathbb{R}^{d \times d}$ contains the projection vectors. The solution of the above optimization problem is given by the generalized eigenvalue problem

$$
\mathbf{X}^{\top} \mathbf{M}^{\prime} \mathbf{X V}=\boldsymbol{\Gamma} \mathbf{X}^{\top} \mathbf{X V} \Leftrightarrow\left(\mathbf{X}^{\top} \mathbf{X}\right)^{-1} \mathbf{X}^{\top} \mathbf{M}^{\prime} \mathbf{X V}=\boldsymbol{\Gamma} \mathbf{V}
$$

where $\boldsymbol{\Gamma}$ is the diagonal matrix of the eigenvalues $\gamma_{i}$ of $\left(\mathbf{X}^{\top} \mathbf{X}\right)^{-1} \mathbf{X}^{\top} \mathbf{M}^{\prime} \mathbf{X}$ and $\mathbf{V}$ is the matrix whose columns contain the eigenvectors $\mathbf{v}_{i}$ of $\mathbf{M}^{\prime} \mathbf{X}$. To enforce the orthogonality constraint $\mathbf{W}^{\top} \mathbf{W}=\mathbf{I}$ each eigenvector $\mathbf{v}_{i}$ must be divided by $\left\|\mathbf{v}_{i}\right\|$ to get normalized eigenvectors $\mathbf{v}_{i}$. The optimal linear projection of the data points to dimension $l=1, \ldots, d$ is given by the first $l$ dominant eigenvectors $\mathbf{v}_{i}, i=1, \ldots, l$, that is, $\mathbf{W}^{*}=\left\{\mathbf{v}_{i}\right\}_{i=1}^{l}$. The matrix of the projected data points is given by $\mathbf{X}^{\mathcal{P}}=\mathbf{X} \mathbf{W}^{*}$. The algorithm to compute the optimal linear projections is described in Algorithm 2.

\section{B. Graph Derivation of KQMI}

We now derive the intrinsic graph that corresponds to the proposed dimensionality reduction algorithm, which is based on the non-parametric MI. In the following we assume that the data in the matrix $\mathbf{X}$ are sorted according to their class

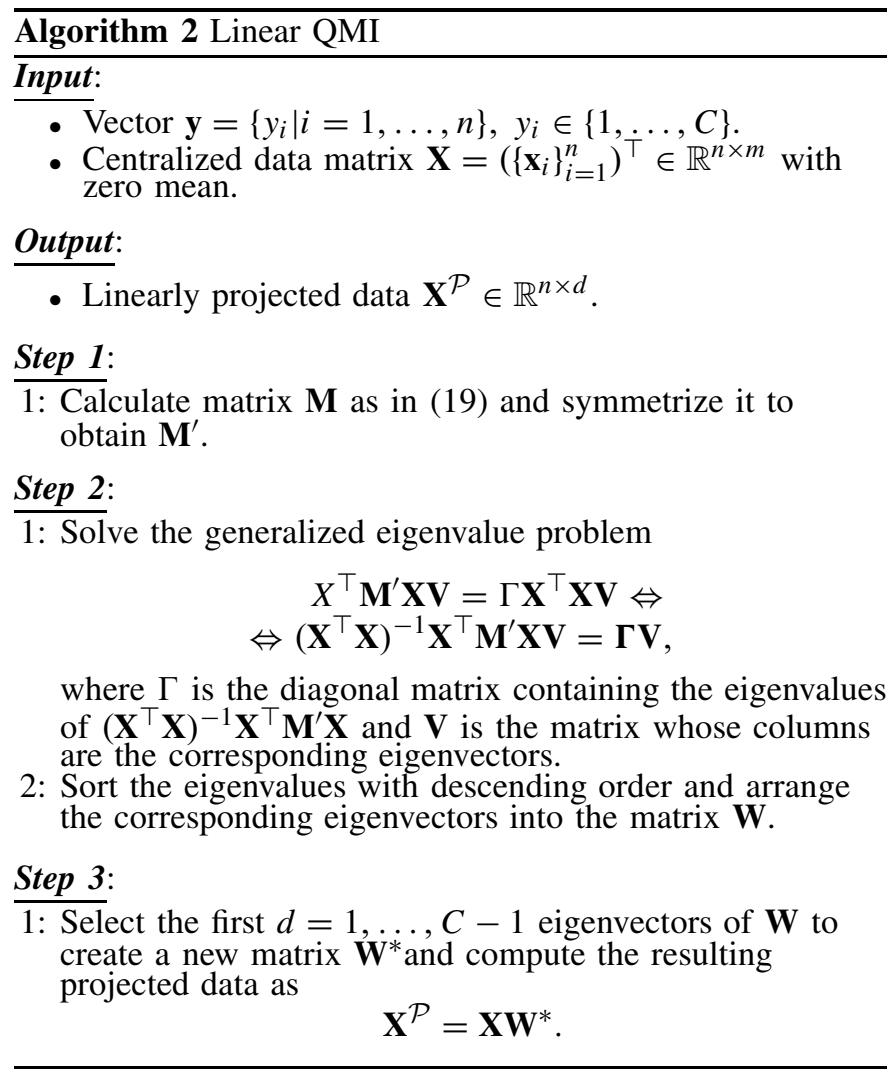

labels. By defining $\alpha_{c}=C_{\mathrm{IN}}+C_{\mathrm{ALL}}-2 C_{\mathrm{BTW}, c}$ and $\beta_{c, c^{\prime}}=$ $C_{\mathrm{ALL}}-C_{\mathrm{BTW}, c}-C_{\mathrm{BTW}, \mathrm{c}^{\prime}}$, the matrix $\mathbf{M}^{\prime}$ has the form

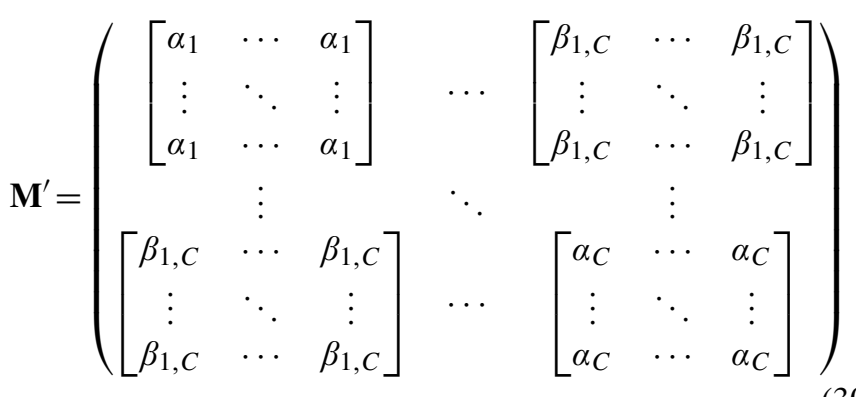

where each submatrix containing the $\alpha_{c}, c=1, \ldots, C$ entries has dimensions $J_{c} \times J_{c}$ and each submatrix containing the $\beta_{c, c^{\prime}}, c=1, \ldots, C, c^{\prime}=1, \ldots, C$ entries has dimensions $J_{c} \times J_{c^{\prime}}$.

By defining a graph with weight matrix $\mathbf{W}$ as

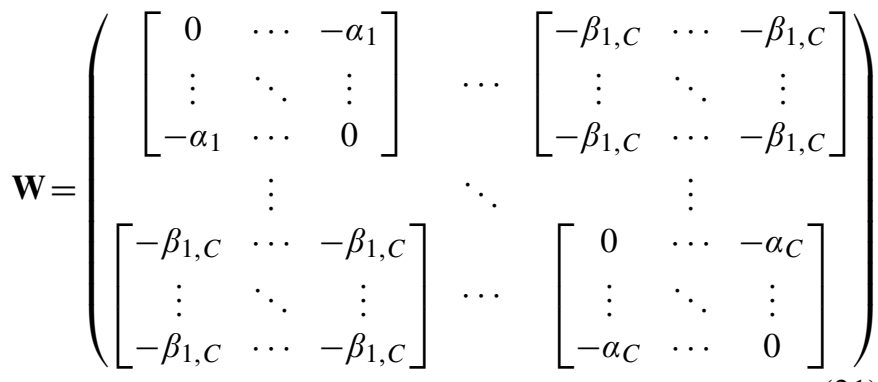

the matrix $\mathbf{M}^{\prime}$ is the Laplacian matrix of this graph and can be written as $\mathbf{M}^{\prime}=\mathbf{D}-\mathbf{W}$, where $\mathbf{D}=\operatorname{diag}\left(\mathbf{M}^{\prime}\right)$. In Appendix $B$, 
it is shown that the property $\sum_{j=1}^{n} W_{i j}=D_{i i}, i=1, \ldots, n$ holds. The above weight matrix $\mathbf{W}$ corresponds to a fully connected graph, where data samples that belong to the same class are connected with edges of nonpositive weights $-\alpha_{c}=2 C_{\mathrm{BTW}, c}-C_{\mathrm{IN}}-C_{\mathrm{ALL}}$ and data samples belonging to different classes are connected with edges of nonnegative weights $-\beta_{c, c^{\prime}}=C_{\mathrm{BTW}, c}+C_{\mathrm{BTW}, c^{\prime}}-C_{\mathrm{ALL}}$.

\section{Discussion}

One of the key contributions of the proposed approach is to interpret the QMI in the graph embedding framework to derive novel closed form dimensionality reduction objectives. To do so, someone has to consider either to maximize the QMI between the samples and the labels after the dimensionality reduction, which is what Torrkola did, giving an iterative algorithm in [33], or to use the information provided by the QMI formulation to build new dimensionality reduction criteria that can be solved in closed form, which is what we propose. If someone wants to follow the second approach he can observe that in the QMI formulation given in (18), we have two elements that define the measure. One is the matrix $\Phi$ that corresponds to the data samples in the feature space and has been derived by a specific kernel used in Parzen estimation (i.e., the Gaussian kernel), to define sample similarity. The other element is the matrix $\mathbf{M}$ in (19) that represents the links between the data samples in the intrinsic graph that forms the QMI measure. Thus, using different kernels in Parzen estimation will result in different forms of $\Phi$ with the same matrix M that defines the graph connections. Moreover, someone can directly use the matrix $\mathbf{M}$ in the graph embedding framework and change the feature map using other well-known kernels. The simplest form of $\Phi$ is the original data matrix $\mathbf{X}$. If we consider that the data samples are the outcome of a projection to lower dimension using $\mathbf{W}$ then the data matrix $\mathbf{X}$ is replaced by $\mathbf{X W}$. Proceeding from the linear case to the nonlinear case, the projection matrix $\mathbf{W}$ is a linear combination of the samples in the feature space given in $\Phi$, that is, $\mathbf{W}=\left\{\mathbf{w}_{i}\right\}_{i=1}^{n}=\left\{\Phi^{\top} \boldsymbol{\alpha}_{i}\right\}_{i=1}^{n}=\Phi^{\top} \mathbf{A} \in \mathbb{R}^{n \times n}$. In all cases, the dimensionality reduction objectives depend on a constant graph embedding matrix $\mathbf{M}$ that represents the QMI sample interactions and a feature matrix $\Phi$ that represents the samples in the corresponding Hilbert space and can be considered to correspond to a specific kernel used in Parzen estimation. Finding the kernel for the Parzen estimation that corresponds to the well-known kernels used in projecting samples in RKHS is out the scope of the paper and will be considered in future research.

For the derivation of KQMI in Section III-A, we enforce an additional constraint of unit covariance of the embedding vectors. This modification was done for two reasons. First, this covariance condition implies that the projected points $\Phi \mathbf{W}$ will be different from each other, because of the orthogonality of the columns of $\Phi \mathbf{W}$ [22]. This is similar to what PCA does. Second, we also conducted experiments without this constraint, however, they were slightly worse than the ones we present in Section IV using the additional constraint.

Furthermore, another logical question arises of why to use the MI as a criterion for dimensionality reduction. It is
TABLE I

BENCHMARK DATASETS' CHARACTERISTICS

\begin{tabular}{|c|c|c|c|c|}
\hline Dataset & Library & Samples & Attributes & Classes \\
\hline Australian & Statlog & 690 & 14 & 2 \\
\hline Balance & UCI & 625 & 4 & 3 \\
\hline Breast Cancer & UCI & 683 & 10 & 2 \\
\hline Dermatology & UCI & 366 & 34 & 6 \\
\hline Diabetes & UCI & 768 & 8 & 2 \\
\hline Ecoli & UCI & 336 & 7 & 8 \\
\hline German Numer & Statlog & 1000 & 24 & 2 \\
\hline Glass & UCI & 214 & 9 & 6 \\
\hline Heart & Statlog & 270 & 13 & 2 \\
\hline Ionosphere & UCI & 351 & 34 & 2 \\
\hline Iris & UCI & 150 & 4 & 3 \\
\hline Liver Disorders & UCI & 214 & 9 & 7 \\
\hline Segment & Statlog & 2310 & 19 & 7 \\
\hline Sonar & UCI & 208 & 60 & 2 \\
\hline Soy & UCI & 307 & 35 & 3 \\
\hline Thyroid & UCI & 215 & 5 & 3 \\
\hline Vehicle & Statlog & 846 & 18 & 4 \\
\hline Vowel & UCI & 990 & 10 & 11 \\
\hline Wine & UCI & 178 & 13 & 3 \\
\hline Zoo & UCI & 101 & 17 & 7 \\
\hline
\end{tabular}

known that the Bayes error rate is the optimal criterion for classification and it can take the form [33]

$$
E(X)=\int_{\mathbf{x}} p(\mathbf{x})\left(1-\max _{i} p\left(y_{i} \mid \mathbf{x}\right)\right) d \mathbf{x} .
$$

The above criterion needs the computation of class posterior probabilities and numerical integration of those. This is a difficult problem given only one training dataset. Several approximations have been proposed that use parametric estimation of class-conditional densities followed by numerical optimization [13], [29]. For example, LDA assumes all classes to be Gaussian with a single shared covariance matrix. The key difference of MI with the already proposed approximations is the fact that it accounts for high-order statistics and not only the second order. Another major property is that MI bounds the Bayes error rate. An upper bound of the form $E(X) \leq(1 / 2)(H(Y)-I(X, Y))$ is given in [16]. Furthermore, in [8], a lower bound involving the Bayes error rate and the MI is proved. Both bounds are minimized when the MI between classes and data points is maximized. That means that we can use the MI as an alternative criterion of the Bayes error rate.

\section{EXPERIMENTAL RESULTS}

In this section, we illustrate the experimental results obtained by comparing our proposed methods against several dimensionality reduction methods available.

\section{A. Experimental Results on Benchmark Datasets}

We compared our proposed dimensionality reduction methods against other competitive methods (i.e., LDA, PCA, supervised LPP, GDA, KCFD, supervised KLPP, and KPCA) using 20 benchmark datasets from the UCI [10], and Statlog [25], repositories. The characteristics of each dataset can be observed in Table I. Let us note here that the methods LPP and KLPP can be either unsupervised or supervised. In this evaluation, we have implemented the supervised versions, since our goal is to compare the supervised KQMI with as many supervised methods as possible. In these versions of 
TABLE II

Classification ERror Rates of Linear and Kernel Dimensionality Reduction Methods With NCC Classifier. In PARENTHESES the Dimensionality of the Final Projected Features Is Shown. The Last Row Shows the Number of Wins for Each Method Across All Datasets

\begin{tabular}{|c|c|c|c|c|c|c|c|c|c|}
\hline & & & Kernel Metho & & & & & Iethed & \\
\hline Dataset & KQMI & GDA & KLPP & CKFD & KPCA & LQMI & LDA & PCA & LPP \\
\hline Australian & $15.51 \%(1)$ & $24.20 \%(1)$ & $16.52 \%(1)$ & $20.44 \%(1)$ & $14.49 \%(14)$ & $14.20 \%(1)$ & $14.20 \%(1)$ & $13.77 \%(4)$ & $14.20 \%(1)$ \\
\hline Balance & $13.12 \%(1)$ & $16.47 \%(2)$ & $13.44 \%(1)$ & $13.74 \%(1)$ & $23.83 \%(4)$ & $25.76 \%(2)$ & $29.89 \%(2)$ & $26.23 \%(4)$ & $25.76 \%(2)$ \\
\hline Breast C & $3.23 \%(1)$ & $4.25 \%(1)$ & $\frac{13.44 \%(1)}{3.66 \%(1)}$ & $\frac{15.14 \%(1)}{3.81 \%(1)}$ & $3.36 \%(4)$ & $3.82 \%(1)$ & $3.82 \%(1)$ & $3.52 \%(1)$ & $3.82 \%(1)$ \\
\hline Derm & $12.84 \%(5)$ & $14.80 \%(5)$ & $14.51 \%(5)$ & $17.53 \%(10)$ & $17.26 \%(34)$ & $2.98 \%(5)$ & $3.53 \%(5)$ & $3.27 \%(17)$ & $2.98 \%(5)$ \\
\hline Diab & (1) & $29.05 \%(1)$ & $26.70 \%(1)$ & $27.22 \%(1)$ & $27.74 \%(7)$ & $\%(1)$ & $23.44 \%(1)$ & $26.83 \%(7)$ & $23.44 \%(1)$ \\
\hline Ecoli & 19.13 & $19.44 \%(7)$ & $19.38 \%(7)$ & $19.67 \%(7)$ & $26.61 \%(5)$ & $22.92 \%(5)$ & $22.09 \%(7)$ & $24.65 \%(7)$ & $22.10 \%(6)$ \\
\hline German Numer & $28.10 \%(1)$ & $28.30 \%(1)$ & $28.30 \%(1)$ & $\%(2)$ & $40.40 \%(23)$ & $27.60 \%(1)$ & $27.60 \%(1)$ & $29.50 \%(22)$ & $27.60 \%(1)$ \\
\hline Glass & & $32.80 \%(5)$ & $29.53 \%(5)$ & $30.42 \%(5)$ & $52.97 \%(8)$ & $38.63 \%(5)$ & $43.60 \%(5)$ & $55.26 \%(8)$ & $.29 \%(5)$ \\
\hline He: & 19.23 & $22.59 \%(1)$ & $22.59 \%(1)$ & $21.11 \%(2)$ & $\%(13)$ & $\%(1)$ & $15.56 \%$ (1) & $18.52 \%$ (11) & $15.56 \%(1)$ \\
\hline Tonos & & $6.52 \%(1)$ & & $6(2)$ & $6(19)$ & $6(1)$ & $16.44 \%$ (1) & $25.27 \%(8)$ & $12.75 \%$ (1) \\
\hline Iris & $2.67 \%$ & $4.00 \%(2)$ & $3.33 \%(2)$ & o(2) & $6.66 \%(4)$ & (1) & $2.00 \%(1)$ & $5.33 \%(1)$ & $2.00 \%$ (1) \\
\hline Liver Disorders & $28.94 \%(1)$ & $32.97 \%(1)$ & $29.50 \%(1)$ & $\%(1)$ & 41.98 & $6(1)$ & $37.12 \%(1)$ & $40.51 \%(6)$ & $37.12 \%(1)$ \\
\hline Segm & $3.72 \%(6)$ & $2.46 \%(6)$ & $4.37 \%(6)$ & $6(6)$ & $15.45 \%$ (19) & $6(6)$ & $35.58 \%(6)$ & $16.02 \%$ (11) & $11.99 \%(5)$ \\
\hline Son & $13.03 \%$ (1) & $12.53 \%(1)$ & $12.53 \%(1)$ & 26.5 & $21.65 \%(40)$ & $24.70 \%(1)$ & $24.22 \%$ (1) & $29.36 \%$ (11) & $24.22 \%(1)$ \\
\hline & $5.23 \%(2)$ & $6.56 \%(2)$ & $5.90 \%(2)$ & 11.17 & $14.04 \%(27)$ & 5.889 & $8.16 \%(2)$ & $22.90 \%(30)$ & $5.88 \%(2)$ \\
\hline Thyl & $3.25 \%$ & $3.70 \%(2)$ & $2.79 \%(2)$ & $3.27 \%(2)$ & $3.70 \%(3)$ & $4.22 \%(1)$ & $4.68 \%(1)$ & $6.52 \%(2)$ & $4.68 \%(1)$ \\
\hline Vehi & $20.32 \%(3)$ & $18.45 \%(3)$ & $21.28 \%(3)$ & $19.75 \%(3)$ & $47.16 \%$ (18) & $21.28 \%(3)$ & $21.28 \%(3)$ & $56.03 \%(15)$ & $22.23 \%(3)$ \\
\hline Vowel & $1.01 \%(10)$ & $0.51 \%(10)$ & $3.43 \%(10)$ & $4.85 \%(8)$ & $41.92 \%(10)$ & $39.29 \%(6)$ & $38.89 \%(6)$ & $49.09 \%(10)$ & $39.39 \%(6)$ \\
\hline Wine & $0.56 \%(2)$ & $0.56 \%(2)$ & $0.56 \%(2)$ & $1.11 \%(2)$ & $1.64 \%(7)$ & $1.67 \%(2)$ & $\frac{5.05 \%(2)}{1.70 \%(2)}$ & $4.45 \%(2)$ & $1.11 \%(2)$ \\
\hline Zoo & $30.63 \%(6)$ & $31.74 \%(6)$ & $30.63 \%(6)$ & $39.01 \%(12)$ & $49.38 \%(15)$ & $24.88 \%(5)$ & $25.79 \%(1)$ & $28.81 \%(5)$ & $25.79 \%(1)$ \\
\hline Rank & 12 & 5 & 6 & 0 & 1 & 12 & 9 & 2 & 11 \\
\hline
\end{tabular}

TABLE III

Classification Error Rates of Linear and Kernel Dimensionality Reduction Methods With KNN Classifier $(K=3)$. In Parentheses the Dimensionality of the Final Projected Features Is Shown. The Last Row Shows THE Number of Wins for Each Method Across All Datasets

\begin{tabular}{|c|c|c|c|c|c|c|c|c|c|}
\hline & \multicolumn{5}{|c|}{ Kernel Methods } & \multicolumn{4}{|c|}{ Linear Methods } \\
\hline Dataset & KQMI & GDA & KLPP & CKFD & KPCA & LQMI & LDA & PCA & LPP \\
\hline Australian & $16.54 \%(1)$ & $25.07 \%(1)$ & $16.25 \%(1)$ & $20.44 \%(1)$ & $15.81 \%(13)$ & $15.21 \%(1)$ & $15.21 \%(1)$ & $15.22 \%(8)$ & $15.94 \%(1)$ \\
\hline Balance & $3.20 \%(1)$ & $8.31 \%(1)$ & $3.52 \%(1)$ & $4.15 \%(1)$ & $12.95 \%(4)$ & $11.20 \%(2)$ & $10.72 \%(2)$ & $20.79 \%(4)$ & $11.52 \%(2)$ \\
\hline Breast Cancer & $3.81 \%(1)$ & $4.11 \%(1)$ & $3.52 \%(1)$ & $3.96 \%(1)$ & $2.78 \%(6)$ & $3.66 \%(1)$ & $3.66 \%(1)$ & $2.64 \%(5)$ & \\
\hline Dermatology & $12.84 \%(5)$ & $14.80 \%(5)$ & $13.95 \%(5)$ & $12.33 \%(10)$ & $10.12 \%(21)$ & $3.28 \%(5)$ & $3.28 \%(5)$ & $2.44 \%(17)$ & $3.82 \%(1)$ \\
\hline Diabetes & $28.38 \%(1)$ & $28.12 \%(1)$ & $27.47 \%(1)$ & $28.13 \%(1)$ & $25.92 \%(8)$ & $25.77 \%(1)$ & $26.82 \%(1)$ & $25.14 \%(7)$ & $27.08 \%(1)$ \\
\hline Ecoli & $18.04 \%(7)$ & $20.53 \%(7)$ & $18.28 \%(7)$ & $18.62 \%(7)$ & $17.21 \%(6)$ & $18.83 \%(3)$ & $18.55 \%(5)$ & $19.67 \%(6)$ & $19.68 \%(5)$ \\
\hline German & $30.00 \%(1)$ & $28.30 \%(1)$ & $29.40 \%(1)$ & $28.60 \%(2)$ & $29.80 \%(21)$ & & $29.80 \%(1)$ & $28.50 \%(21)$ & $29.30 \%(1)$ \\
\hline German & $31.64 \%(5)$ & $35.26 \%(5)$ & $31.69 \%(5)$ & $32.58 \%(6)$ & $\frac{2.00 \%(21)}{34.49 \%(6)}$ & $34.06 \%(5)$ & $36.36 \%(3)$ & $\frac{20.000 \%(21)}{29.92 \%(5)}$ & $39.30 \%(5)$ \\
\hline & 18.89\% (1) & $\frac{3.20 \%(1)}{22.59 \%(1)}$ & $\frac{31.09 \%(1)}{21.85 \%(1)}$ & $\frac{32.50 \%(0)}{20.00 \%(2)}$ & $\frac{34.49 \%(0)}{23.33 \%(3)}$ & $\frac{3.00 \%(1)}{18.89 \%(1)}$ & $18.89 \%(1)$ & $\frac{29.92 \%(5)}{20.00 \%(6)}$ & $\mathbf{1 8 . 8 9 \% ( 1 )}$ \\
\hline Ionosphere & & $6.51 \%(1)$ & $5.97 \%(1)$ & $7.08 \%(2)$ & $4.81 \%(9)$ & $14.22 \%(1)$ & $18.98 \%(1)$ & $10.76 \%$ (11) & $14.78 \%(1)$ \\
\hline Iris & & $3.33 \%(2)$ & $2.67 \%(2)$ & $4.67 \%(2)$ & $\%(3)$ & $\%(1)$ & $2.00 \%(1)$ & $4.67 \%(4)$ & $2.00 \%(1)$ \\
\hline Liver Di & 34.5 & & $34.77 \%(1)$ & 33.00 & $\%(5)$ & & $0 \%(1)$ & $35.35 \%(6)$ & $38.60 \%(1)$ \\
\hline Segm & 2.90 & $2.68 \%(6)$ & $2.94 \%(10)$ & (6) & $\%(19)$ & 3.0 & $7.84 \%(5)$ & $3.59 \%$ (11) & $3.85 \%(6)$ \\
\hline Son: & 12.53 & $12.53 \%(1)$ & $12.53 \%(1)$ & $12.56 \%(2)$ & $25.49 \%(13)$ & (1) & $22.29 \%(1)$ & $12.46 \%(21)$ & $22.29 \%(1)$ \\
\hline Soy & & (2) & $8.81 \%(2)$ & & $10.44 \%(28)$ & & $7.17 \%(2)$ & $5.56 \%(27)$ & $5.55 \%(2)$ \\
\hline Thyroid & & & $2.79 \%$ & & $2.32 \%(3)$ & & $3.72 \%(2)$ & $2.81 \%(1)$ & $3.72 \%(2)$ \\
\hline Vehi & $19.75 \%(3)$ & & $17.85 \%(3)$ & $18.34 \%(4)$ & $33.80 \%$ (18) & $\%(3)$ & $23.43(3)$ & $29.66 \%(15)$ & $24.61 \%(3)$ \\
\hline Vowel & $0.61 \%(10)$ & $0.40 \%(10)$ & $2.02 \%(10)$ & $2.12 \%(9)$ & $4.85 \%(9)$ & $3.23 \%(9)$ & $3.03 \%(10)$ & $2.83 \%(10)$ & $3.43 \%(9)$ \\
\hline Wine & $1.67 \%(2)$ & $0.56 \%(2)$ & $0.56 \%(2)$ & $1.11 \%(2)$ & $1.67 \%(5)$ & $1.11 \%(2)$ & $1.11 \%(2)$ & $1.70 \%(7)$ & $1.11 \%(2)$ \\
\hline Zoo & $30.63 \%(6)$ & $31.74 \%(6)$ & $39.63 \%(6)$ & $31.74 \%(12)$ & $35.36 \%(10)$ & $24.88 \%(5)$ & $25.79 \%(1)$ & $26.90 \%(4)$ & $25.79 \%(1)$ \\
\hline Rank & 7 & 6 & 4 & 2 & 6 & 8 & 8 & 9 & 3 \\
\hline
\end{tabular}

the algorithms, we compute the $K$ nearest neighbors (KNNs) graph by connecting with edges the points that belong to the same class and they are among the KNNs of each other.

All the features of each dataset were scaled to the interval $[-1,+1]$. To evaluate the test error on the different experiments, we used 10 -fold cross validation. In each fold, we first compute the eigenvectors for each method based only on the fold's training set; and then, we project the feature vectors of the fold's training and test sets on the acquired eigenvectors. As classifiers we used the nearest class centroid (NCC) classifier, the KNNs classifier [12] with $K=3$, and the LIBSVMs [5] SVM classifier with linear and radial basis function (RBF) kernels. We set the cost variable $C$ for both linear and RBF SVM to $C=100$ and for the RBF SVM we set the RBF kernel's $\sigma$ to $\sigma=1$. For the kernel dimensionality reduction methods, we used RBF kernel with $\sigma=1$. The experimental results for the NCC classifier can be observed in Table II, those of the KNN classifier can be observed in Table III, for the linear SVM classifier in Table IV and for the
RBF SVM classifier in Table V. In each column, we illustrate the minimum classification error attained by each classifier for the specific dimensionality reduction method and in the parentheses the number of dimensions this error has been achieved. In the last row of each one of these tables we can see the rankings (i.e., the number of winning datasets) of each dimensionality reduction method.

In the case of the NCC classifier (i.e., Table II), we can see that KQMI obtains better performance than the other kernel methods. In the case of the linear projection algorithms, the LQMI method displays comparable results with the supervised LPP method, while both methods seem to outperform LDA. For the KNN classifier (i.e., Table III), we can see that KQMI and GDA display almost the same performance, obtaining though better results than CKFD and KLPP. It is interesting to note here the relatively good performance of the KPCA method, which for the KNN classifier wins the same number of datasets as the supervised GDA method. For the same classifier and for the linear projection algorithms the LQMI 
TABLE IV

Classification ERror Rates of Linear and Kernel Dimensionality Reduction Methods With Linear KeRnel SVM Classifier $(C=100)$. In Parentheses the Dimensionality of the Final Projected Features Is Shown. The Last Row Shows the Number of Wins for Each Method Across All Datasets

\begin{tabular}{|c|c|c|c|c|c|c|c|c|c|}
\hline & \multicolumn{5}{|c|}{ Kernel Methods } & \multicolumn{4}{|c|}{ Linear Methods } \\
\hline Dataset & KQMI & GDA & KLPP & CKFD & KPCA & LQMI & LDA & PCA & LPP \\
\hline Australian & $15.22 \%(1)$ & $24.20 \%(1)$ & $16.38 \%(1)$ & $20.44 \%(1)$ & $14.78 \%(14)$ & $14.20 \%(1)$ & $14.20 \%(1)$ & $14.49 \%(4)$ & $14.20 \%(1)$ \\
\hline Balance & $3.68 \%(1)$ & $10.08 \%(1)$ & $3.84 \%(1)$ & $3.68 \%(1)$ & $8.95 \%(4)$ & $8.32 \%(1)$ & $8.32 \%(2)$ & $8.32 \%(4)$ & $8.32 \%(1)$ \\
\hline Breast Cancer & $3.23 \%(1)$ & $4.10 \%(1)$ & $3.66 \%(1)$ & $4.10 \%(1)$ & $3.23 \%(1)$ & $3.23 \%(1)$ & $3.23 \%(1)$ & $2.64 \%(5)$ & $3.23 \%(1)$ \\
\hline Dermatology & $12.84 \%(5)$ & $14.80 \%(5)$ & $13.95 \%(5)$ & $13.67 \%(10)$ & $11.17 \%(33)$ & $3.28 \%(5)$ & $3.27 \%(5)$ & $1.36 \%(21)$ & $3.26 \%(5)$ \\
\hline Diabetes & $22.79 \%(1)$ & $31.25 \%(1)$ & $26.18 \%(1)$ & $27.09 \%(1)$ & $22.40 \%(8)$ & $23.32 \%(1)$ & $23.32 \%(1)$ & $23.45 \%(7)$ & $23.32 \%(1)$ \\
\hline Ecoli & $19.10 \%(7)$ & $26.06 \%(7)$ & $19.36 \%(6)$ & $20.50 \%(7)$ & $22.10 \%(6)$ & $19.89 \%(6)$ & $20.17 \%(6)$ & $20.17 \%(7)$ & $20.18 \%(6)$ \\
\hline German Numer & $27.70 \%(1)$ & $28.30 \%(1)$ & $28.00 \%(1)$ & $28.20 \%(2)$ & $28.90 \%(24)$ & $23.10 \%(1)$ & $23.10 \%(1)$ & $23.00 \%(24)$ & $23.10 \%(1)$ \\
\hline Glass & $31.69 \%(5)$ & $46.89 \%(5)$ & $30.80 \%(4)$ & $30.40 \%(5)$ & $35.14 \%(7)$ & $35.99 \%(1)$ & $36.94 \%(5)$ & $36.19 \%(7)$ & $37.83 \%(5)$ \\
\hline Heart & $20.00 \%(1)$ & $22.59 \%(1)$ & $21.48 \%(1)$ & $20.37 \%$ (1) & $20.00 \%(6)$ & $15.93 \%$ (1) & $15.93 \%(1)$ & $15.93 \%(12)$ & $15.93 \%(1)$ \\
\hline Tonosphere & $5.08 \%(1)$ & $6.53 \%(1)$ & $5.69 \%(1)$ & $6.49 \%(1)$ & $6.54 \%(28)$ & $13.31 \%(1)$ & $35.89 \%(1)$ & $10.22 \%(19)$ & $13.03 \%(1)$ \\
\hline Iris & $3.33 \%(2)$ & $4.00 \%(2)$ & $3.33 \%(2)$ & $4.00 \%(2)$ & $4.00 \%(2)$ & $3.33 \%(1)$ & $2.67 \%(1)$ & $4.67 \%(4)$ & $3.33 \%(1)$ \\
\hline Liver Disorders & $27.49 \%$ (1) & $42.01 \%(1)$ & $28.64 \%(1)$ & $30.66 \%$ (1) & $42.02 \%(1)$ & $31.33 \%(1)$ & $30.75 \%(1)$ & $30.76 \%(6)$ & $31.04 \%$ (1) \\
\hline Segment & $3.16 \%(6)$ & $2.51 \%(6)$ & $3.38 \%(6)$ & $3.77 \%(6)$ & $7.45 \%(13)$ & $5.46 \%(6)$ & $85.71 \%(1)$ & $4.29 \%(13)$ & $5.93 \%(6)$ \\
\hline Sonar & $12.53 \%(1)$ & $12.53 \%(1)$ & $13.03 \%(1)$ & $12.56 \%(2)$ & $25.49 \%(13)$ & $24.22 \%(1)$ & $24.22 \%(1)$ & $19.73 \%(1)$ & $23.75 \%(1)$ \\
\hline Soy & $5.89 \%(2)$ & $5.56 \%(1)$ & $4.59 \%(2)$ & $6.22 \%(3)$ & $19.56 \%(33)$ & $5.88 \%(2)$ & $12.02 \%(2)$ & $3.58 \%(30)$ & $5.88 \%(2)$ \\
\hline Thyroid & $1.86 \%(2)$ & $8.33 \%(2)$ & $2.31 \%(2)$ & $1.88 \%(2)$ & $2.32 \%(3)$ & $3.72 \%(2)$ & $3.72 \%(2)$ & $2.79 \%(1)$ & $3.27 \%(1)$ \\
\hline Vehicle & $19.85 \%(3)$ & $18.45 \%(3)$ & $16.80 \%(3)$ & $18.34 \%(4)$ & $33.80 \%(18)$ & $21.29 \%(3)$ & $21.16 \%(3)$ & $19.40 \%$ (18) & $21.05 \%(03)$ \\
\hline Vowel & $0.61 \%(10)$ & $0.51 \%(10)$ & $2.73 \%(10)$ & $2.12 \%(9)$ & $4.84 \%(9)$ & $19.60 \%(10)$ & $19.29 \%(10)$ & $18.88 \%(10)$ & $19.70 \%(10)$ \\
\hline Wine & $1.11 \%(2)$ & $0.56 \%(2)$ & $0.56 \%(2)$ & $1.11 \%(3)$ & $1.64 \%(5)$ & $1.70 \%(2)$ & $1.11(2)$ & $1.70 \%(5)$ & $2.26 \%(2)$ \\
\hline Zoo & $30.63 \%(6)$ & $31.74 \%(6)$ & $30.63 \%(6)$ & $30.63 \%(12)$ & $38.15 \%(12)$ & $24.89 \%(5)$ & $25.79 \%(1)$ & $26.90 \%(4)$ & $25.79 \%(3)$ \\
\hline Rank & 11 & 4 & 5 & 3 & 4 & 7 & 7 & 12 & 4 \\
\hline
\end{tabular}

TABLE V

Classification ERror Rates of Linear And KeRnel Dimensionality Reduction Methods With RBF KeRnel SVM Classifier $(C=100, \sigma=1)$. In Parentheses the Dimensionality of the Final Projected Features is Shown.

The Last Row Shows the Number of Wins for Each Method Across All Datasets

\begin{tabular}{|c|c|c|c|c|c|c|c|c|c|}
\hline & \multicolumn{5}{|c|}{ Kernel Methods } & \multicolumn{4}{|c|}{ Linear Methods } \\
\hline Dataset & KQMI & GDA & KLPP & CKFD & KPCA & LQMI & LDA & PCA & LPP \\
\hline Australian & $15.08 \%(1)$ & $24.10 \%(1)$ & $16.24 \%(1)$ & $20.43 \%(2)$ & $19.85 \%(1)$ & $13.47 \%(1)$ & $13.77 \%(1)$ & $14.50 \%(4)$ & $13.32 \%(1)$ \\
\hline Balance & $3.20 \%(1)$ & $10.08 \%(1)$ & $3.67 \%(1)$ & $4.48 \%(1)$ & $31.37 \%(3)$ & $8.32 \%(1)$ & $8.32 \%(1)$ & $5.27 \%(4)$ & $8.32 \%(1)$ \\
\hline Breast Cancer & $3.37 \%(1)$ & $4.11 \%(1)$ & $3.66 \%(1)$ & $3.96 \%(1)$ & $3.37 \%$ (1) & $2.78 \%(1)$ & $3.66 \%(1)$ & $2.64 \%(5)$ & $2.78 \%(1)$ \\
\hline Dermatology & $12.84 \%(5)$ & $14.80 \%(5)$ & $13.95 \%(5)$ & $21.30 \%(5)$ & $16.64 \%(4)$ & $3.28 \%(5)$ & $12.32 \%(5)$ & $5.20 \%(7)$ & $3.28 \%(5)$ \\
\hline Diabetes & $22.79 \%(1)$ & $28.65 \%(1)$ & $26.05 \%(1)$ & $27.22 \%(1)$ & $33.86 \%(8)$ & $23.84 \%(1)$ & $23.57 \%(1)$ & $25.14 \%(7)$ & $23.58 \%(1)$ \\
\hline Ecoli & $19.10 \%(7)$ & $22.16 \%(7)$ & $19.93 \%(7)$ & $29.04 \%(3)$ & $41.15 \%(2)$ & $19.09 \%(7)$ & $26.33 \%(6)$ & $19.13 \%(7)$ & $19.89 \%(7)$ \\
\hline German Numer & $27.90 \%(1)$ & $28.30 \%(1)$ & $28.70 \%(1)$ & $28.50 \%(2)$ & $30.30 \%(23)$ & $22.60 \%(1)$ & $22.90 \%(1)$ & $28.40 \%(21)$ & $22.60 \%(1)$ \\
\hline Glass & $32.21 \%(5)$ & $32.31 \%(5)$ & $30.35 \%(4)$ & $34.31 \%(5)$ & $52.30 \%(6)$ & $34.67 \%(4)$ & $37.17 \%(3)$ & $26.99 \%(5)$ & $36.94 \%(4)$ \\
\hline Heart & $19.26 \%(1)$ & $22.59 \%(1)$ & $23.33 \%(1)$ & $20.00 \%(2)$ & $23.33 \%(3)$ & $15.92 \%(1)$ & $18.89 \%(1)$ & $20.00 \%(6)$ & $16.30 \%(1)$ \\
\hline Ionosphere & $5.36 \%(1)$ & $6.81 \%(1)$ & $5.97 \%(1)$ & $7.10 \%(2)$ & $22.14 \%(3)$ & $13.05 \%(1)$ & $35.89 \%(1)$ & $5.38 \%(12)$ & $13.05 \%(1)$ \\
\hline Iris & $3.33 \%(2)$ & $4.00 \%(2)$ & $3.33 \%(2)$ & $6.67 \%(2)$ & $8.67 \%(3)$ & $3.33 \%(1)$ & $2.67 \%(1)$ & $5.33 \%(4)$ & $3.33 \%(1)$ \\
\hline Liver Disorders & $27.47 \%(1)$ & $40.85 \%(1)$ & $28.64 \%(1)$ & $31.24 \%(1)$ & $38.81 \%(4)$ & $30.47 \%(1)$ & $30.75 \%(1)$ & $29.22 \%(6)$ & $30.47 \%(1)$ \\
\hline Segment & $2.86 \%(6)$ & $2.51 \%(6)$ & $3.33 \%(6)$ & $6.23 \%(4)$ & $18.18 \%(3)$ & $3.59 \%(6)$ & $85.71 \%(1)$ & $2.99 \%(11)$ & $5.10 \%(6)$ \\
\hline Sonar & $16.46 \%(1)$ & $12.53 \%(1)$ & $13.03 \%(1)$ & $13.01 \%(2)$ & $36.57 \%(4)$ & $24.22 \%(1)$ & $30.81 \%(1)$ & $13.87 \%(10)$ & $23.75(1)$ \\
\hline Soy & $5.90 \%(2)$ & $6.23 \%(2)$ & $4.92 \%(2)$ & $9.16 \%(3)$ & $32.51 \%(3)$ & $5.57 \%(2)$ & $11.71 \%(2)$ & $5.57 \%(9)$ & $5.56 \%(2)$ \\
\hline Thyroid & $2.32 \%(2)$ & $4.18 \%(2)$ & $2.79 \%(2)$ & $1.86 \%(2)$ & $7.81 \%(2)$ & $3.72 \%(2)$ & $3.72 \%(2)$ & $2.79 \%(1)$ & $3.72 \%$ (1) \\
\hline Vehicle & $19.97 \%(3)$ & $18.33 \%(3)$ & $16.80 \%(3)$ & $19.62 \%(4)$ & $53.80 \%(3)$ & $21.76 \%(3)$ & $27.34(3)$ & $19.87 \%(17)$ & $20.81 \%(3)$ \\
\hline Vowel & $0.61 \%(10)$ & $0.51 \%(10)$ & $2.42 \%(10)$ & $6.46 \%(5)$ & $55.05 \%(3)$ & $1.01 \%(10)$ & $2.02 \%(8)$ & $0.40 \%(10)$ & $1.01 \%(10)$ \\
\hline Wine & $1.11 \%(2)$ & $0.56 \%(2)$ & $0.56 \%(2)$ & $2.22 \%(3)$ & $4.38 \%(2)$ & $1.11 \%(2)$ & $1.11 \%(2)$ & $1.11 \%(13)$ & $1.67 \%(2)$ \\
\hline Zoo & $30.63 \%(6)$ & $31.74 \%(6)$ & $30.63 \%(6)$ & $46.10 \%(3)$ & $48.63 \%(8)$ & $25.79 \%(5)$ & $25.79 \%$ (1) & $28.81 \%(3)$ & $25.79 \%(2)$ \\
\hline Rank & 11 & 4 & 6 & 1 & 1 & 6 & 4 & 11 & 5 \\
\hline
\end{tabular}

and LDA exhibit the same performance winning, however, less sets than the unsupervised PCA. These poor results for the linear supervised methods can be mainly attributed to the inadequate number of samples per class, or due to the fact that the training data might nonuniformly sample the underlying distribution [24]. Moreover, PCA achieves, in most of the cases, better performance in higher dimensions than the supervised methods which are constrained, due to rank deficiency issues, to less than $C-1$ dimensions. Another reason for these good results for the unsupervised PCA method might be the Vapnik-Chervonenkis dimension (VC) [34] of the classifier in use. That is, the powerful KNN and RBF SVM classifiers with high $\mathrm{VC}$ dimension seem to favor more the unsupervised PCA than the other supervised methods. This does not hold for the weaker NCC classifier that seems to favor more the supervised approaches. In Tables IV and V, we can see that for the kernel methods KQMI becomes the dominant method when the SVM classifier is used. For the same classifier but for the linear Methods, we can see again the previously mentioned superiority of the PCA method against the supervised ones.

\section{B. Visualization}

We also conducted visualization experiments, where we used the artificially created Swiss Roll dataset and a subset of 1000 samples from the MNIST handwritten digits dataset. The Swiss Roll dataset was created to test out various dimensionality reduction algorithms. The idea that lies behind the creation of this dataset is to create several points in $\mathbb{R}^{2}$, and then map them to $\mathbb{R}^{3}$ with some smooth function. The resulting 3-D dataset can then be used to test how well a dimensionality method maps the 3-D manifold back to the 2-D space. Here, our purpose is to visualize how well the classes of each dataset are separated in the 2-D projective space. For the Swiss Roll, the 2-D projections of the kernel dimensionality reduction methods (i.e., KQMI, GDA, supervised KLPP, CKFD, and KPCA) can be observed in Fig. 1, and those of the linear dimensionality reduction methods 


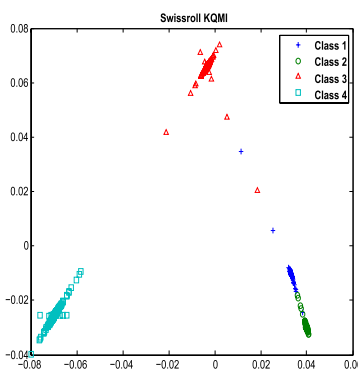

(a)

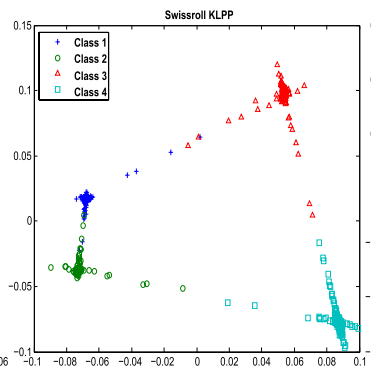

(b)

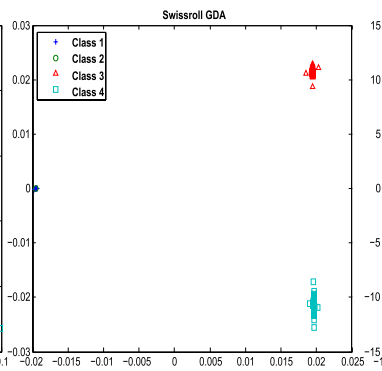

(c)

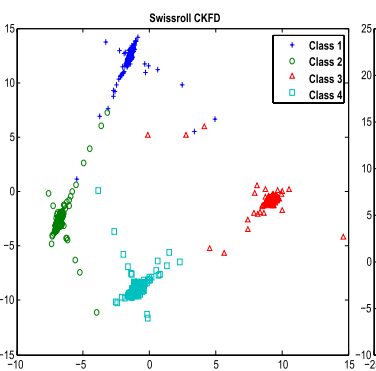

(d)

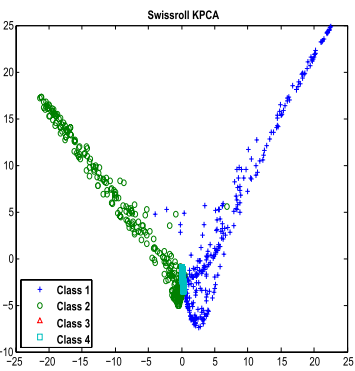

(e)

Fig. 1. 2-D projections of the Swiss Roll dataset using kernel dimensionality reduction methods. (a) KQMI. (b) KLPP. (c) GDA. (d) CKFD. (e) KPCA.

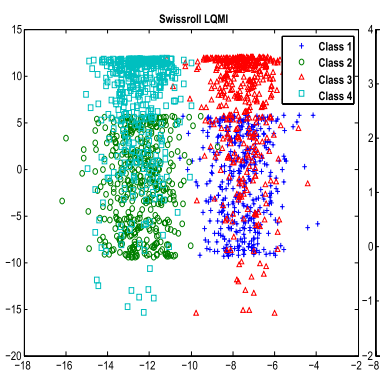

(a)

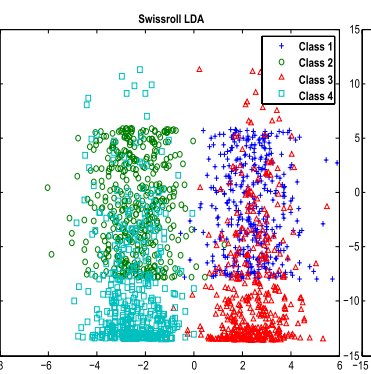

(b)

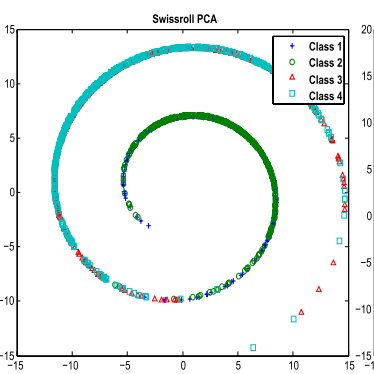

(c)

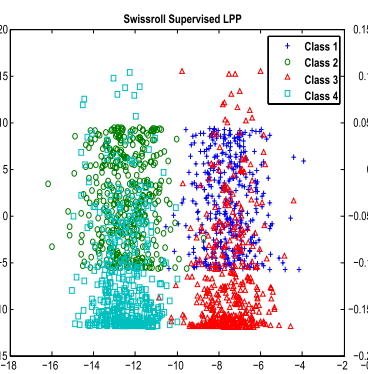

(d)

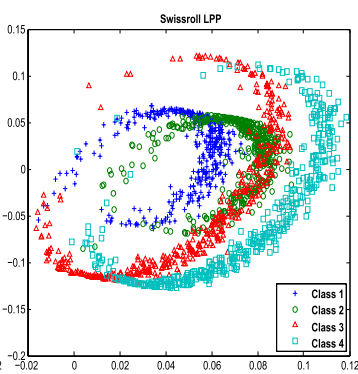

(e)

Fig. 2. 2-D projections of the Swiss Roll dataset using linear dimensionality reduction methods. (a) LQMI. (b) LDA. (c) PCA. (d) Supervised LPP. (e) LPP.

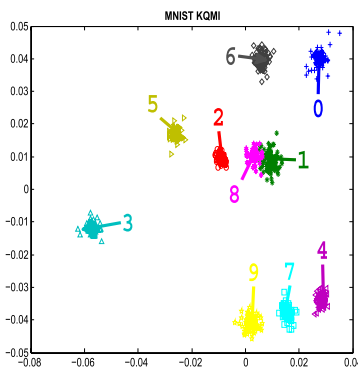

(a)

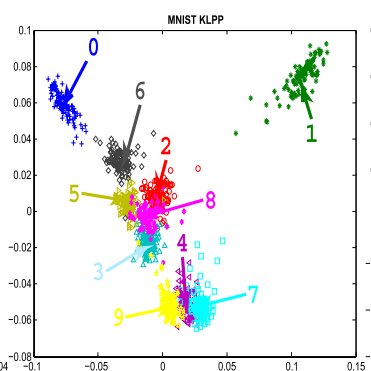

(b)

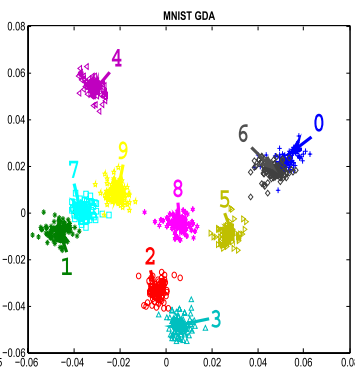

(c)

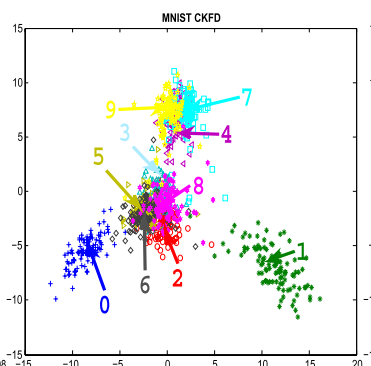

(d)

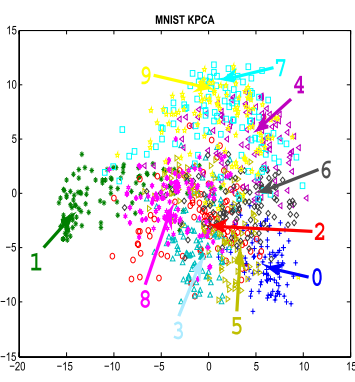

(e)

Fig. 3. 2-D projections of the MNIST dataset using kernel dimensionality reduction methods. (a) KQMI. (b) KLPP. (c) GDA. (d) CKFD. (e) KPCA.

(i.e., LQMI, LDA, PCA, and supervised LPP) can be observed in Fig. 2.

Comparing Figs. 1 and 2, we observe that the linear methods fail to achieve a mapping in which the dataset's classes are well separated in the 2-D space, while the supervised kernel methods (i.e., KQMI, GDA, KLPP, and CKFD) give a relatively good mapping in terms of class separability. In this setting, GDA performs best with KQMI and KLPP giving similar results, even though KQMI produces more compact clusters with less linearly nonseparable points than KLPP. Moreover, from the above figures we can verify that the unsupervised methods, both kernel and linear, fail to give a good mapping in which the classes are well separated, a fact to be expected due to their unsupervised nature.

In Figs. 3 and 4, we can see the 2-D visualization of the MNIST dataset with the kernel and linear reduction methods, respectively. We can see how nicely the KQMI and GDA methods produce a 2-D projection subspace, in which the classes are very well separated, with the KQMI attaining a better separation of the classes than that of the GDA. It could
TABLE VI

FACE RECOGNITION AND FACIAL EXPRESSION RECOGNITION DATASETS' ChaRACTERISTICS

\begin{tabular}{|c|c|c|c|}
\hline Dataset & Samples & Attributes & Classes \\
\hline ORL & 400 & 1024 & 40 \\
\hline YALE & 165 & 1024 & 15 \\
\hline JAFFE & 213 & 1200 & 7 \\
\hline KANADE & 704 & 1200 & 7 \\
\hline
\end{tabular}

be counter-intuitive the fact that the distance between digits 1 and 8 is much smaller than that between 1 and 7 . However, let us note here that the objective function considers all the classes simultaneously and thus, if a specific projection worsens the separability between two specific classes, whereas it enhances the separability among all the other classes, it is expected to be selected as an optimal projection. In the specific case, we can see that indeed the separability of all the classes is enhanced compared with the one obtained with the KLPP projections except the separability between digits 1 and 8 which is worse. That is, the separability between 1 and 8 


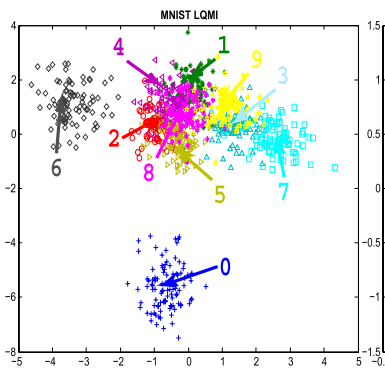

(a)

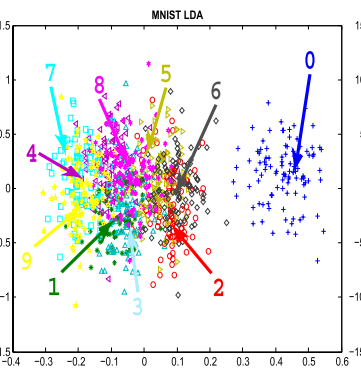

(b)

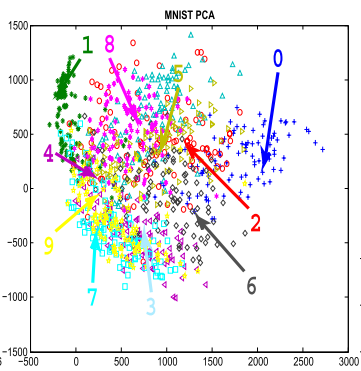

(c)

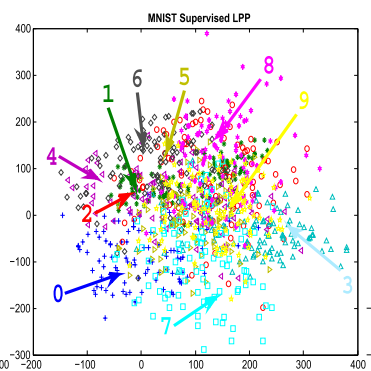

(d)

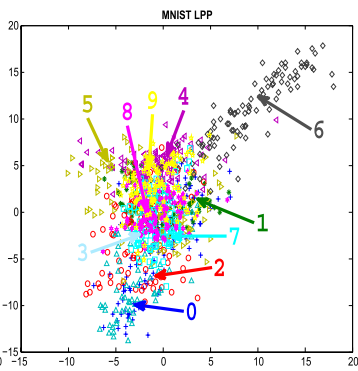

(e)

Fig. 4. 2-D projections of the MNIST dataset using linear dimensionality reduction methods. (a) LQMI. (b) LDA. (c) PCA. (d) Supervised LPP. (e) LPP.

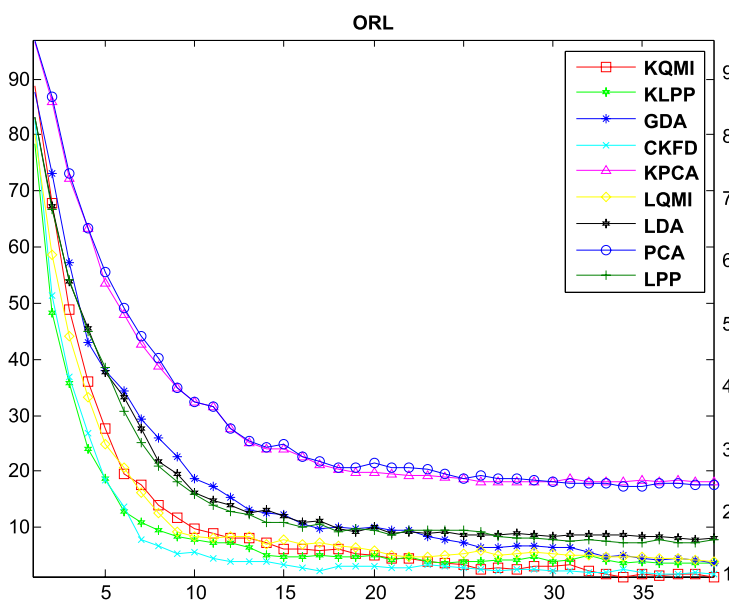

(a)

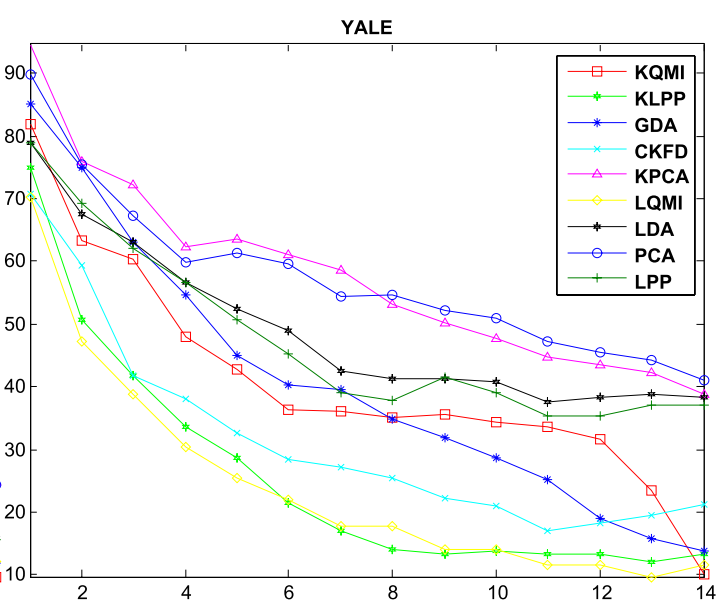

(b)

Fig. 5. Results on (a) ORL and (b) YALE face recognition datasets. The horizontal axis shows the dimension of the projected features for several dimensions. The vertical axis shows the corresponding classification error rate.

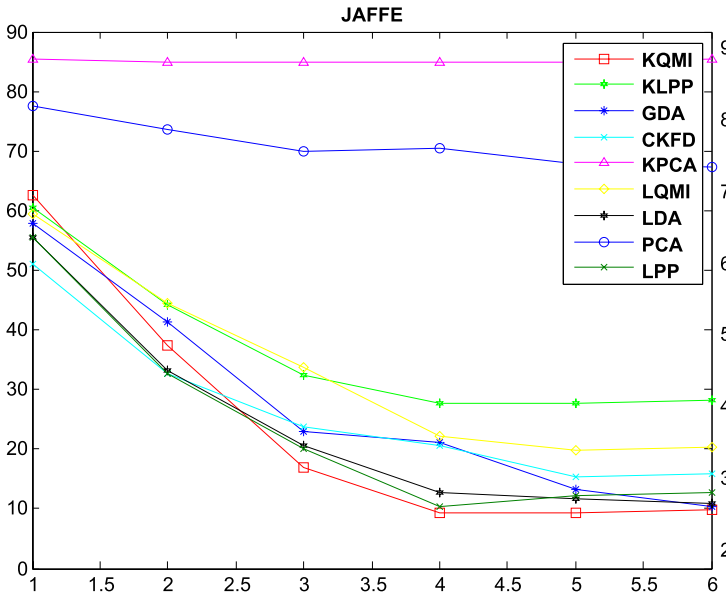

(a)

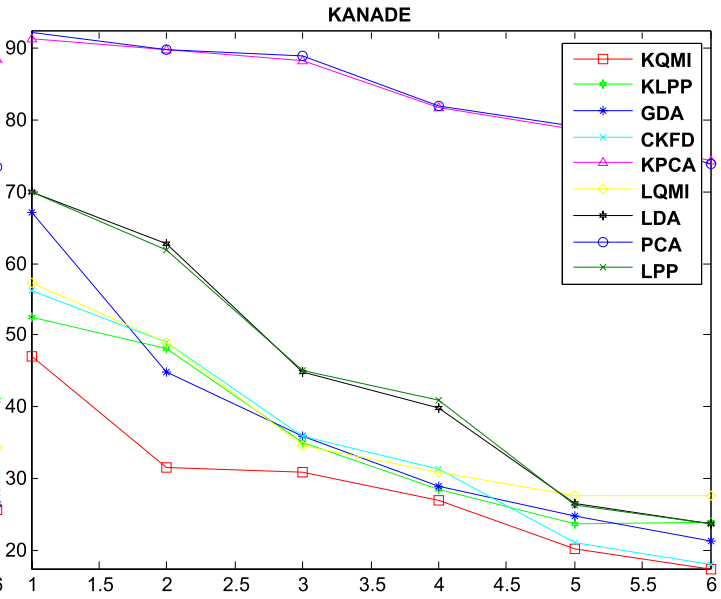

(b)

Fig. 6. Results on (a) JAFFE and (b) KANADE facial expression recognition datasets. The horizontal axis shows the dimension of the projected features for several dimensions. The vertical axis shows the corresponding classification error rate.

is the prize to be payed to enhance the other 44 pairwise separabilities. The situation changes in the cases of the CKFD and KLPP methods, where as we can see although we can moderately discriminate relatively good the classes, many of them seem to coincide. All the linear methods fail to give a 2-D projection where the classes are well separated. However, we can mention here that LQMI gives fairly better 2-D projection in terms of class discrimination than all the other linear methods.

\section{Face Recognition and Facial Expression Recognition}

We also tested our methods on two face recognition (i.e., ORL and YALE) and two facial expression recognition datasets (i.e., JAFFE and KANADE). The characteristics of each dataset can be observed in Table VI.

We normalized all the feature vectors of each dataset to unit length and for the linear methods only, we preprocessed the datasets with PCA to hold $99 \%$ of the initial dataset 
TABLE VII

Classification Error Rates of Linear and Kernel Dimensionality Reduction Methods With NCC Classifier. In Parentheses the Dimensionality of the Final Projected Features Is Shown

\begin{tabular}{|c|c|c|c|c|c|c|c|c|c|}
\hline & \multicolumn{5}{|c|}{ Kernel Methods } & \multicolumn{4}{|c|}{ Linear Methods } \\
\hline Dataset & KQMI & GDA & KLPP & CKFD & KPCA & LQMI & LDA & PCA & LPP \\
\hline "ORL & $1.25 \%(34)$ & $3.75 \%(39)$ & $3.50 \%(24)$ & $1.75 \%(36)$ & $18.00 \%(28)$ & $4.00 \%(39)$ & $7.75 \%(38)$ & $214.00 \%(142)$ & $7.25 \%(34)$ \\
\hline YALE & $10.22 \%(14)$ & $12.14 \%(14)$ & $13.39 \%(14)$ & $17.01 \%(11)$ & $27.71 \%$ & $9.74 \%(13)$ & $37.65 \%(11)$ & $29.63 \%(67)$ & $35.34 \%(12)$ \\
\hline JAFFE & $9.29 \%(4)$ & $10.37 \%(6)$ & $27.78 \%(4)$ & $15.34 \%(5)$ & $48.48 \%(148)$ & $19.76 \%(5)$ & $10.81 \%(6)$ & $52.20 \%(93)$ & $10.31 \%(4)$ \\
\hline KANADE & $17.46 \%(6)$ & $21.27 \%(6)$ & $23.73 \%(5)$ & $17.88 \%(12)$ & $48.30 \%(130)$ & $24.67 \%(6)$ & $23.69 \%(6)$ & $46.02 \%(175)$ & $23.71 \%(6)$ \\
\hline
\end{tabular}

TABLE VIII

Classification ERror Rates of Linear and Kernel Dimensionality Reduction Methods With KNN Classifier $(K=3)$. In Parentheses the Dimensionality of the Final Projected Features Is Shown

\begin{tabular}{|c|c|c|c|c|c|c|c|c|c|}
\hline & \multicolumn{5}{|c|}{ Kernel Methods } & \multicolumn{4}{|c|}{ Linear Methods } \\
\hline Dataset & KQMI & GDA & KLPP & CKFD & KPCA & LQMI & LDA & PCA & LPP \\
\hline ORL & $1.50 \%(38)$ & $.75 \%(39)$ & & $150 \%(36)$ & $1000 \%(45)$ & $6.50 \%(35)$ & $8.25 \%(26$ & $8.50 \%$ & $6.50 \%(34)$ \\
\hline YALE & $2.55 \%(14)$ & $3.91 \%(14)$ & $9.16 \%(1$ & $17.12 \%$ & $35.52 \%$ & $35.95 \%$ & $37.72 \%$ & $20.07 \%$ & $11.24 \%(4)$ \\
\hline JAFFE & $8.92 \%(6)$ & $10.37 \%(6)$ & $15.01 \%(5)$ & $13.95 \%(12)$ & $15.07 \%(86)$ & $11.78 \%(13)$ & $10.81 \%(6)$ & $15.03 \%(14)$ & $11.24 \%(4)$ \\
\hline KANADE & $17.32 \%(6)$ & $21.27 \%(6)$ & $20.46 \%(6)$ & $17.76 \%(12)$ & $42.03 \%(17)$ & $23.98 \%(6)$ & $24.69 \%(6)$ & $42.32 \%(18)$ & $25.12 \%(6)$ \\
\hline
\end{tabular}

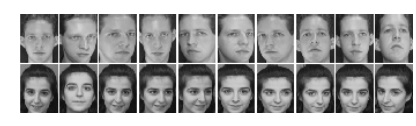

Fig. 7. Two subjects from the ORL face recognition dataset.

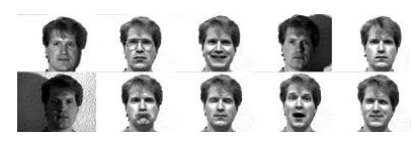

Fig. 8. Subject from the YALE face recognition dataset.

variance. As can be observed in Table VI, for all the datasets the number of the dataset samples $n$ is less than the number of dataset attributes $m$. This situation is known as the undersampled size problem. For datasets, where the under-sampled size problem exists, in many cases occur singularities that in turn result in very bad performance of the eigenvalue analysis algorithm. One of the solutions to overcome the under-sampled size problem is to perform an initial PCA step on the data and fall to the dimension, where its associated eigenvalue is greater than some threshold [37]. For the evaluation of the generalization error, we used the same procedure as in Section IV-A. In the sequel, we give the characteristics of the real-life datasets used in our evaluation.

1) $O R L$ [28]: The dataset contains 40 individuals and 10 different images for each individual, including variations in facial expression and pose. In Fig. 7, we can see a male and a female subject from the ORL dataset.

2) YALE [2]: The dataset contains 165 gray-scale images of 15 individuals. There are 11 images per subject, one per different facial expression or configuration: center-light, with glasses, happy, left-light, without glasses, normal, right-light, sad, sleepy, surprised, and wink. Fig. 8 shows a subject from the YALE dataset.

3) JAFFE [23]: The dataset contains 213 images of seven facial expressions (six basic facial expressions+ one neutral) posed by 10 Japanese female models. Each image has been labeled with one of six emotion adjectives (i.e., fear, anger, disgust, happiness, surprise, and sadness). In Fig. 9, we see a subject from the Japanese Female Facial Expression Database (JAFFE) dataset.

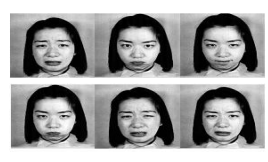

Fig. 9. Subject from the JAFFE facial expression recognition dataset.

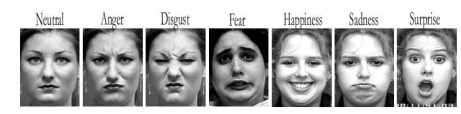

Fig. 10. Various subjects from the KANADE facial expression recognition dataset.

4) KANADE [19]: The dataset contains 704 images of seven facial expressions (six basic facial expressions+ one neutral). Each image has been rated on six emotion adjectives (i.e., fear, anger, disgust, happiness, surprise, and sadness). The variety of subjects covers different races, ages, and genders. The database is collected under controlled illumination and background. In Fig. 10, various subjects from the KANADE dataset are given.

In Tables VII and VIII, we show the classification errors on the above mentioned datasets attained by the various dimensionality reduction methods using the NCC and KNN classifiers, respectively. We also conducted experiments using as classifiers the linear and RBF SVM. However, for these classifiers the dominant method was PCA, and all the remaining methods attained equivalent performance. Therefore, we omit the results of these experiments.

In Fig. 5(a) and (b), we illustrate the diagrams of the classification error using the NCC classifier in the dimensions $1-(C-1)$ for the ORL and YALE datasets, respectively. In Fig. 6(a) and (b), we can see the resulting classification errors using the NCC classifier on the JAFFE and KANADE datasets attained in the dimensions $1-(C-1)$ as well.

From the results in Table VII, we can see that, in the case of the NCC classifier, KQMI obtains better performance in most of the datasets than all the other kernel methods. In the linear case for the NCC classifier, the LQMI wins more datasets than the other methods. For the KNN classifier in Table VIII, we can see that both KQMI and LQMI exhibit slightly worse 
performance than that attained for the NCC classifier, winning however, most of the datasets.

In Figs. 5 and 6, we also see that our proposed methods, in most of the cases, converge faster to their best performance compared with the other methods and hence, they display better performance in dimensions lower than $C-1$.

\section{CONCLUSion}

In this paper, we proposed a novel supervised dimensionality reduction method based on the maximization of a nonparametric MI criterion between the feature vectors and their respective class labels. We formulated the QMI as a kernel objective function that can be directly optimized inside the graph embedding framework. We also derived the linear equivalent of this kernel method, and we compared both methods to several state-of-the-art kernel and linear dimensionality reduction methods. From the experimental results, we can conclude that the proposed methods obtain comparable and in most cases even better classification accuracy than the stateof-the-art.

\section{APPENDIX A \\ MI TERMS}

In this appendix, we derive analytically the expressions for the $V_{\mathrm{ALL}}, V_{\mathrm{IN}}$, and $V_{\mathrm{BTW}}$ terms. We have

$$
\begin{aligned}
V_{\mathrm{ALL}} & =\frac{1}{n^{2}}\left(\sum_{c=1}^{C}\left(\frac{J_{c}}{n}\right)^{2}\right) \sum_{j=1}^{n} \sum_{k=1}^{n} \mathbf{K}_{j k}=\left(\frac{1}{n^{4}} \sum_{c=1}^{C}\left(J_{c}\right)^{2}\right) \mathbf{1}^{\top} \mathbf{K} \mathbf{1} \\
& =C_{\mathrm{ALL}} \mathbf{1}^{\top} \mathbf{K} \mathbf{1}=C_{\mathrm{ALL}} \operatorname{tr}\left\{\mathbf{K 1 1}^{\top}\right\}=C_{\mathrm{ALL}} \operatorname{tr}\left\{\Phi \Phi^{\top} \mathbf{1 1}^{\top}\right\} \\
& =C_{\mathrm{ALL}} \operatorname{tr}\left\{\Phi^{\top} \mathbf{1 1}^{\top} \Phi\right\} \\
V_{\mathrm{IN}} & =\frac{1}{n^{2}} \sum_{c=1}^{C} \sum_{j, y_{j}=c} \sum_{k, y_{k}=c} \mathbf{K}_{j k}=C_{\mathrm{IN}} \sum_{c=1}^{C} \mathbf{1}_{c}^{\top} \mathbf{K} \mathbf{1}_{c} \\
& =C_{\mathrm{IN}} \sum_{c=1}^{C} \operatorname{tr}\left\{\mathbf{K} \mathbf{1}_{c} \mathbf{1}_{c}^{\top}\right\}=C_{\mathrm{IN}} \sum_{c=1}^{C} \operatorname{tr}\left\{\Phi \Phi^{\top} \mathbf{1}_{c} \mathbf{1}_{c}^{\top}\right\} \\
& =C_{\mathrm{IN}} \operatorname{tr}\left\{\sum_{c=1}^{C} \Phi \Phi^{\top} \mathbf{1}_{c} \mathbf{1}_{c}^{\top}\right\}=C_{\mathrm{IN}} \operatorname{tr}\left\{\Phi \Phi^{\top}\left(\sum_{c=1}^{C} \mathbf{1}_{c} \mathbf{1}_{c}^{\top}\right)\right\} \\
& =C_{\mathrm{IN}} \operatorname{tr}\left\{\Phi^{\top}\left(\sum_{c=1}^{C} \mathbf{1}_{c} \mathbf{1}_{c}^{\top}\right) \Phi\right\}
\end{aligned}
$$$$
V_{\mathrm{BTW}}=\frac{1}{n^{3}} \sum_{c=1}^{C} J_{c} \sum_{k, y_{k}=c} \sum_{j=1}^{n} \mathbf{K}_{k j}=\sum_{c=1}^{C} C_{\mathrm{BTW}, c} \mathbf{1}_{c}^{\top} \mathbf{K} \mathbf{1}
$$$$
=\sum_{c=1}^{C} \operatorname{tr}\left\{C_{\mathrm{BTW}, c} \mathbf{K} \mathbf{1 1}_{c}^{\top}\right\}=\sum_{c=1}^{C} \operatorname{tr}\left\{C_{\mathrm{BTW}, c} \Phi \Phi^{\top} \mathbf{1 1}_{c}^{\top}\right\}
$$$$
=\operatorname{tr}\left\{\sum_{c=1}^{C} C_{\mathrm{BTW}, c} \Phi \Phi^{\top} \mathbf{1 1}_{c}^{\top}\right\}
$$$$
=\operatorname{tr}\left\{\Phi \Phi^{\top} \mathbf{1}\left(\sum_{c=1}^{C} C_{\mathrm{BTW}, c} \mathbf{1}_{c}^{\top}\right)\right\}
$$$$
=\operatorname{tr}\left\{\Phi^{\top} \mathbf{1}\left(\sum_{c=1}^{C} C_{\mathrm{BTW}, c} \mathbf{1}_{c}^{\top}\right) \Phi\right\} \text {. }
$$

\section{APPENDIX B \\ QMI GRAPH FORMULATION}

In this appendix, we show that $\sum_{j=1}^{n} W_{i j}=D_{i i}$, $i=1, \ldots, n$ for the QMI graph

$$
\begin{aligned}
& \left(1-J_{c}\right) \alpha_{c}-\sum_{\substack{c^{\prime}=1, c^{\prime} \neq c}}^{C} \beta_{c, c^{\prime}} J_{c^{\prime}}=\left(1-J_{c}\right)\left(C_{\mathrm{IN}}+C_{\mathrm{ALL}}-2 C_{\mathrm{BTW}, c}\right) \\
& -\sum_{\substack{c^{\prime}=1, c^{\prime} \neq c}}^{C}\left(C_{\mathrm{ALL}}-C_{\mathrm{BTW}, c}-C_{B T W, c^{\prime}}\right) J_{c^{\prime}} \\
& =\alpha_{c}-J_{c} C_{\mathrm{IN}}-J_{c} C_{\mathrm{ALL}}+2 J_{c} C_{\mathrm{BTW}, c} \\
& -\sum_{\substack{c^{\prime}=1, c^{\prime} \neq c}}^{C} C_{\mathrm{ALL}} J_{c^{\prime}}+\sum_{\substack{c^{\prime}=1, c^{\prime} \neq c}}^{C} C_{\mathrm{BTW}, c} J_{c^{\prime}}+\sum_{\substack{c^{\prime}=1, c^{\prime} \neq c}}^{C} C_{B T W, c^{\prime}} J_{c^{\prime}} \\
& =\alpha_{c}-\frac{J_{c}}{n^{2}}-J_{c} C_{\mathrm{ALL}}+2 \frac{J_{c}^{2}}{n^{3}}-C_{\mathrm{ALL}}\left(n-J_{c}\right) \\
& +C_{\mathrm{BTW}, c}\left(n-J_{c}\right)+\sum_{\substack{c^{\prime}=1, c^{\prime} \neq c}}^{C} \frac{J_{c^{\prime}}^{2}}{n^{3}} \\
& =\alpha_{c}-\frac{J_{c}}{n^{2}}-J_{c} C_{\mathrm{ALL}}+2 \frac{J_{c}^{2}}{n^{3}}-n C_{\mathrm{ALL}}+J_{c} C_{\mathrm{ALL}} \\
& +n C_{\mathrm{BTW}, c}-J_{c} C_{\mathrm{BTW}, c}+\sum_{\substack{c^{\prime}=1, c^{\prime} \neq c}}^{n_{c}} \frac{J_{c^{\prime}}^{2}}{n^{3}} \\
& =\alpha_{c}-\frac{J_{c}}{n^{2}}-J_{c} C_{\mathrm{ALL}}+2 \frac{J_{c}^{2}}{n^{3}}-n C_{\mathrm{ALL}}+J_{c} C_{\mathrm{ALL}} \\
& +\frac{J_{c}}{n^{2}}-\frac{J_{c}^{2}}{n^{3}}+\sum_{\substack{c^{\prime}=1, c^{\prime} \neq c}}^{C} \frac{J_{c^{\prime}}^{2}}{n^{3}} \\
& =\alpha_{c}+\frac{J_{c}^{2}}{n^{3}}-n C_{\mathrm{ALL}}+\sum_{\substack{c^{\prime}=1, c^{\prime} \neq c}}^{C} \frac{J_{c^{\prime}}^{2}}{n^{3}}=\alpha_{c}-n C_{\mathrm{ALL}} \\
& +\sum_{c^{\prime}=1}^{C} \frac{J_{c^{\prime}}^{2}}{n^{3}}=\alpha_{c}-n C_{\mathrm{ALL}}+n C_{\mathrm{ALL}}=\alpha_{c} .
\end{aligned}
$$

\section{REFERENCES}

[1] G. Baudat and F. Anouar, "Generalized discriminant analysis using a kernel approach," Neural Comput., vol. 12, no. 10, pp. 2385-2404, 2000.

[2] P. N. Belhumeur, J. P. Hespanha, and D. J. Kriegman, "Eigenfaces vs. Fisherfaces: Recognition using class specific linear projection," IEEE Trans. Pattern Anal. Mach. Intell., vol. 19, no. 7, pp. 711-720, Jul. 1997.

[3] M. Belkin and P. Niyogi, "Laplacian eigenmaps for dimensionality reduction and data representation," Neural Comput., vol. 15, no. 6, pp. 1373-1396, Jun. 2003.

[4] M. L. Braun, J. Buhmann, and K.-R. Müller, "Denoising and dimension reduction in feature space," in Advances in Neural Information Processing Systems 19, B. Schölkopf, J. Platt, and T. Hoffman, Eds. Cambridge, MA, USA: MIT Press, 2007, pp. 185-192.

[5] C.-C. Chang and C.-J. Lin, "LIBSVM: A library for support vector machines," ACM Trans. Intell. Syst. Technol., vol. 2, no. 3, pp. 27:127:27, 2011.

[6] C. Cortes and V. Vapnik, "Support-vector networks," Mach. Learn., vol. 20 , no. 3 , pp. $273-297,1995$. 
[7] T. M. Cover and J. A. Thomas, Elements of Information Theory (Wiley Series in Telecommunications and Signal Processing), 2nd ed. New York, NY, USA: Wiley, Jul. 2006.

[8] R. M. Fano, Transmission of Information: A Statistical Theory of Communications. Cambridge, MA, USA: MIT Press, 1961.

[9] R. A. Fisher, "The use of multiple measurements in taxonomic problems," Ann. Eugenics, vol. 7, no. 2, pp. 179-188, 1936.

[10] K. Bache and M. Lichman. (2013). UCI Machine Learning Repository. School of Inform. and Comput. Sci., University of California, Irvine, CA, USA [Online]. Available: http://archive.ics.uci.edu/ml

[11] K. Fukumizu, F. R. Bach, and M. I. Jordan, "Dimensionality reduction for supervised learning with reproducing kernel Hilbert spaces," J. Mach. Learn. Res., vol. 5, pp. 73-99, Jan. 2004.

[12] K. Fukunaga, Introduction to Statistical Pattern Recognition, 2nd ed. San Francisco, CA, USA: Academic, Oct. 1990.

[13] X. Guorong, C. Peiqi, and W. Minhui, "Bhattacharyya distance feature selection," in Proc. 13th Int. Conf. Pattern Recognit., vol. 2. 1996 pp. 195-199.

[14] S. W. Hadley, F. Rendl, and H. Wolkowicz, "Symmetrization of nonsymmetric quadratic assignment problems and the Hoffman-Wielandt inequality," in Proc. 6th Haifa Conf. Matrix Theory, 1996.

[15] X. He and P. Niyogi, "Locality preserving projections," in Advances in Neural Information Processing Systems 16, S. Thrun, L. Saul, and B. Schölkopf, Eds. Cambridge, MA, USA: MIT Press, 2004.

[16] M. E. Hellman and J. Raviv, "Probability of error, equivocation, and the Chernoff bound," IEEE Trans. Inf. Theory, vol. 16, no. 4, pp. 368-372, Jul. 1970.

[17] T. Hofmann, B. Schölkopf, and A. J. Smola, "Kernel methods in machine learning," Ann. Statist., vol. 36, no. 3, pp. 1171-1220, Jun. 2008.

[18] I. Jolliffe, Principal Component Analysis (Encyclopedia of Statistics in Behavioral Science). New York, NY, USA: Wiley, Oct. 2005.

[19] T. Kanade, Y. Tian, and J. F. Cohn, "Comprehensive database for facial expression analysis," in Proc. 4th IEEE Int. Conf. Autom. Face Gesture Recognit., Mar. 2000, pp. 46-53.

[20] J. Kapur, Measures of Information and their Applications. New York, NY, USA: Wiley, 1994

[21] J. Kapur and H. Kesavan, Entropy Optimization Principles with Applications. San Francisco, CA, USA: Academic, 1992.

[22] E. Kokiopoulou and Y. Saad, "Orthogonal neighborhood preserving projections: A projection-based dimensionality reduction technique," IEEE Trans. Pattern Anal. Mach. Intell., vol. 29, no. 12, pp. 2143-2156, Dec. 2007.

[23] M. J. Lyons, S. Akamatsu, M. Kamachi, and J. Gyoba, "Coding facial expressions with Gabor wavelets," in Proc. 3rd IEEE Int. Conf. Autom. Face Gesture Recognit., Nara, Japan, Apr. 1998, pp. 200-205.

[24] A. M. Martinez and A. C. Kak, "PCA versus LDA," IEEE Trans. Pattern Anal. Mach. Intell., vol. 23, no. 2, pp. 228-233, Feb. 2001.

[25] D. Michie, D. J. Spiegelhalter, and C. C. Taylor, Machine Learning, Neural and Statistical Classification (Ellis Horwood Series in Artificial Intelligence). Englewood Cliffs, NJ, USA: Prentice-Hall, 1994.

[26] S. Mika, G. Ratsch, J. Weston, B. Schölkopf, and K. R. Müller, "Fisher discriminant analysis with kernels," in Proc. 9th IEEE Signal Process. Soc. Workshop Neural Netw. Signal Process., Aug. 1999, pp. 41-48.

[27] S. T. Roweis and L. K. Saul, "Nonlinear dimensionality reduction by locally linear embedding," Science, vol. 290, no. 5500, pp. 2323-2326, 2000.

[28] F. Samaria and A. C. Harter, "Parameterisation of a stochastic model for human face identification," in Proc. 2nd IEEE Workshop Appl. Comput. Vis., Sarasota, FL, USA, Dec. 1994, pp. 138-142.

[29] G. Saon and M. Padmanabhan, "Minimum Bayes error feature selection for continuous speech recognition," in Advances in Neural Information Processing Systems. Cambridge, MA, USA: MIT Press, 2001, pp. 800-806.

[30] B. Schölkopf, A. Smola, and K.-R. Müller, "Nonlinear component analysis as a kernel eigenvalue problem," Neural Comput., vol. 10, no. 5, pp. 1299-1319, 1998

[31] B. Schölkopf and A. J. Smola, Learning With Kernels Support Vector Machines, Regularization, Optimization, and Beyond. Cambridge, MA, USA: MIT Press, Dec. 2001.

[32] J. B. Tenenbaum, V. de Silva, and J. C. Langford, "A global geometric framework for nonlinear dimensionality reduction," Science, vol. 290, no. 5500, pp. 2319-2323, 2000.

[33] K. Torkkola, "Feature extraction by non parametric mutual information maximization," J. Mach. Learn. Res., vol. 3, pp. 1415-1438, Mar. 2003.

[34] V. N. Vapnik, Statistical Learning Theory. New York, NY, USA: Wiley, 1998.
[35] S. Yan, D. Xu, B. Zhang, H.-J. Zhang, Q. Yang, and S. Lin, "Graph embedding and extensions: A general framework for dimensionality reduction," IEEE Trans. Pattern Anal. Mach. Intell., vol. 29, no. 1, pp. 40-51, Jan. 2007.

[36] J. Yang, A. F. Frangi, J.-Y. Yang, D. Zhang, and Z. Jin, "KPCA plus LDA: A complete kernel fisher discriminant framework for feature extraction and recognition," IEEE Trans. Pattern Anal. Mach. Intell., vol. 27, no. 2, pp. 230-244, Feb. 2005.

[37] J. Yang and J.-Y. Yang, "Why can LDA be performed in PCA transformed space?" Pattern Recognit., vol. 36, no. 2, pp. 563-566, 2003.

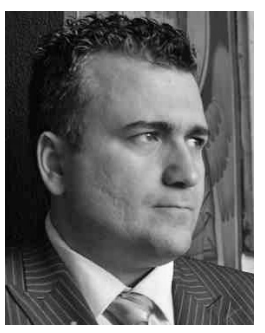

Dimitrios Bouzas received the B.Sc. degree in informatics and the M.Sc. (Hons.) degree in digital media from the Aristotle University of Thessaloniki, Thessaloniki, Greece, in 2010 and 2012, respectively.

$\mathrm{He}$ is currently a Researcher and Developer with the Geometry Division, Beta CAE Systems S.A., Epanomi, Greece. His current research interests include machine learning, computational geometry, computer graphics, numerical analysis, mesh generation algorithms, computational fluid dynamics, stress analysis, and computer-aided engineering.

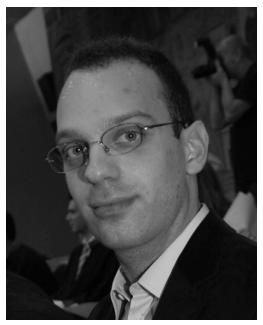

Nikolaos Arvanitopoulos (S'08) received the B.Sc. degree in informatics from the Aristotle University of Thessaloniki, Thessaloniki, Greece, in 2009, and the M.Sc. degree in visual computing from Saarland University, Saabrucken, Germany, in 2011. He is currently pursuing the Ph.D. degree with the Image and Visual Representation Group, School of Computer and Communication Sciences, École Polytechnique Fédérale de Lausanne, Lausanne, Switzerland. His current research interests include machine learning, image processing, and handwriting

recognition

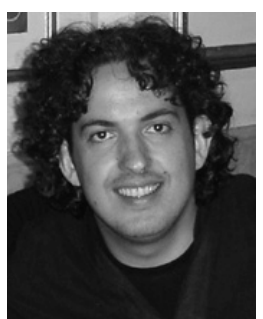

Anastasios Tefas (M'04) received the B.Sc. degree in informatics and the Ph.D. degree in informatics from the Aristotle University of Thessaloniki, Thessaloniki, Greece, in 1997 and 2002, respectively.

$\mathrm{He}$ was a Lecturer with the Aristotle University of Thessaloniki from 2008 to 2012. From 2006 to 2008, he was an Assistant Professor with the Department of Information Management, Technological Institute of Kavala, Didymoteicho, Greece. From 2003 to 2004, he was a temporary Lecturer with the Department of Informatics, University of Thessaloniki. From 1997 to 2002, he was a Researcher and Teaching Assistant with the Department of Informatics, University of Thessaloniki. He has been an Assistant Professor with the Department of Informatics, Aristotle University of Thessaloniki, since 2013. He participated in 12 research projects financed by national and European funds. He has co-authored 42 journal papers, 122 papers in international conferences, and contributed seven chapters to edited books in his area of expertise, with the Google Scholar citations of over 2220, and an H-index of 24. His current research interests include computational intelligence, pattern recognition, statistical machine learning, digital signal and image processing, and computer vision. 